\title{
Structure in Context: A Morphological View of Whole Network Performance
}

\author{
K. Dennie Kim \\ kimd@darden.virginia.edu \\ Darden School of Business \\ University of Virginia \\ Russell J. Funk \\ rfunk@umn.edu \\ Akbar Zaheer \\ azaheer@umn.edu \\ Carlson School of Management \\ University of Minnesota
}

\begin{abstract}
Network perspectives in organizational research have focused primarily on how the embeddedness of actors shapes individual, or nodal, outcomes. Against this backdrop, a growing number of researchers have begun to adopt a wider lens on organizational networks, shifting the focus to collective, or whole network, performance. Yet, efforts to understand the relationship between whole network structure and whole network performance have produced conflicting findings, which suggests that a different approach may be needed. Drawing on macrostructural sociology, we propose a "whole network morphology" framework, which argues the whole network structure-performance relationship is contingent on other fundamental—relational and cultural—whole network dimensions. Subsequently, we undertake an application of our framework, through which we demonstrate how a morphological view helps address conflicting findings on the structure-performance relationship. Leveraging data on 350 million physician relationships, we study 250 whole networks known as Accountable Care Organizations (ACOs). Consistent with previous work, we do not find a clear association between structural connectedness and performance. However, we find that a more disconnected network structure is associated with negative ACO performance when the relational strength of network ties is high. We also find evidence of better ACO performance in the presence of a physician cultural orientation when the whole network is more connected.
\end{abstract}


Research on organizational networks has typically focused on understanding how the embeddedness of actors shapes their outcomes (Burt 1992, Uzzi 1997, Rowley et al. 2000). Against this backdrop, however, a growing group of scholars have adopted a fundamental perspective shift, moving the lens away from nodal outcomes towards whole network performance (e.g. Rosenkopf and Schilling 2007, Fang et al. 2010, Guler and Nerkar 2012). This work examines how the overall pattern of ties among a group of actors shapes their collective outcomes. Put differently, unlike "ego" network studies, this literature uses whole network structure to predict whole network performance, a contrast shown in Figure 1. Taken together, studies in this stream offer evidence that the best-performing whole network structures are not those that maximize individual network members' performance (Mason and Watts 2012, Shore et al. 2015) but rather-because of spillovers across nodes, emergent properties of complex systems, and similar factors-are those that help network members function well as a whole (McCubbins et al. 2009). Thus, findings from the whole network literature suggest that the mechanisms underpinning whole network performance are different from those of ego networks.

[Figure 1 here]

Notwithstanding this progress, literature on whole network performance has produced conflicting findings, which makes it difficult to cumulate insights across studies. Notably, scholars disagree over the relationship between whole network structure-in particular, "connectedness," i.e., the extent to which network members can reach one another through direct or indirect paths (Lazer and Friedman 2007, Centola 2010, Funk 2014)—and whole network outcomes. One stream argues that the most effective whole networks are those that achieve coordination through disconnected structures, where individual members interact with only a few others (Lazer and Friedman 2007, Derex and Boyd 2016). Although disconnectedness may reduce the speed of information flow through a network, it is also theorized to promote greater diversity of ideas (Fang et al. 2010, Derex and Boyd 2016), reduced noise (Christie et al. 1952), and fewer redundancies (Enemark et al. 2011). Other studies have argued the opposite, instead suggesting that greater connectedness is preferable (Fleming et al. 2007). This work finds that more connected structures facilitate communication 
(Kearns et al. 2006), expose actors to a greater variety of peers (Mason and Watts 2012, Enemark et al. 2014), and may help with conflict management (McCubbins et al. 2009). To date, scholars have attempted to address these conflicting findings by examining whether the structure-performance relationship in whole networks is contingent on the task being undertaken by network members. However, studies have also observed networks with radically different structures producing similar outcomes when conducting the same tasks (Lazer and Friedman 2007, Fang et al. 2010, Mason and Watts 2012). Thus, understanding of the relationship between whole network structure and performance remains limited.

In this paper, we address this gap in understanding by proposing a novel theoretical framework, supported by an empirical application. Drawing on macrostructural sociology—a literature that has developed in isolation from work on whole networks-we expand research on whole networks beyond its focus on structure to include other network dimensions. We build on Blau's (1960 1964) work, which theorized the emergence of (macro) structures from individual (micro) interactions, taking the position that structure is not a given, nor a constant. Instead, because structure is an emergent feature of social activity, its topology depends on the overall norms and values that influence interacting actors and the nature of the relationships they form. We extend this logic to argue that even though the same structural pattern may be observed in different networks (Milo et al. 2002), the relationship between whole network structure and performance is inherently contingent upon the other whole network dimensions.

Based on the assertion that whole network structure does not uniquely determine whole network performance, we propose a framework of whole network morphology, inspired by the study of relationships among biological structures. We draw particularly on biomechanical morphology, which posits that "single traits should not be studied in isolation... [because] the performance consequences of a particular morphological change can depend on other aspects of an [organism]" (Koehl, 1996: 515). As an example, Koehl (1996: 515) highlights that the coincidence of multiple "flyer traits" in flying frogs "improved turning performance significantly more than expected from the sum of their individual effects." Our morphology framework argues similarly on the relationship between whole 
network structure and performance- the same whole network structures, when viewed in conjunction with other dimensions, may be associated with different behaviors and thus outcomes. Accordingly, our morphological view emphasizes the fundamental contingency of the relationship between whole network structure and performance.

The origins of these contingencies are at the theoretical core of the network morphology framework. By adapting and extending Blau's theories of macrostructure, we argue that the contingencies that affect the structure-performance relationship are in fact other emergent dimensions — the relational and cultural dimensions_ of whole networks. Moreover, in our morphological view of whole network performance, the structural dimension, itself an emergent property, would not exist in the absence of the other dimensions. Therefore, considering the structure-performance relationship in the context of other dimensions may provide insight into why whole networks with similar structures may exhibit different outcomes.

We argue that a novel whole network-level framework is necessary because the application of ego-level theories to whole networks may lead to erroneous predictions. ${ }^{1}$ Specifically, there is a "lack of parallel meaning" (Lazersfeld 1993) between many ego-level or nodal constructs and those at the whole network level, in that such constructs do not aggregate, or disaggregate, easily across levels. Consider the concept of embeddedness (Granovetter 1985), in which the behavior (and outcomes) of individual actors is shaped by their social ties (Rowley et al. 2000, Seibert et al. 2001, Moran 2005).2 The embeddedness of a node lacks a parallel network-level construct because unlike a node- the unit of analysis in ego network research—the whole network— the unit of analysis in whole network research—cannot embed itself—“the boundary of a boundary is zero" (Wheeler 1990). The distinction between (ego level) centrality and (network level) centralization offers another useful

\footnotetext{
${ }^{1}$ Macrostructural sociologists have long cautioned against applying concepts developed at one level to phenomenon at a different level. Paralleling our arguments, for example, Blau (1986: vii, vx) writes, "Macrosociological and microsociological analysis involve different theoretical approaches and employ different concepts...the distinctive problems of nations or cities cannot be clarified by dissecting...interpersonal relations but only by analyzing the implications for social life of major structural features." Similarly, we argue that whole networks cannot be understood using frameworks developed to understand ego networks.

2 This view of embeddedness as a tool for explaining the outcomes of individuals (rather than whole networks) is evident in Granovetter (1985: 487, emphasis added), who writes, "Actors do not behave or decide as atoms outside a social context...Their attempts at purposive action are instead embedded in...social relations."
} 
illustration. Research on ego networks commonly finds that actors with greater centrality (i.e., better positional embeddedness) experience better outcomes (Ibarra 1993, Tsai 2001). The primary mechanisms reflect an inflow of resources to ego based on his or her favorable position. However, at the whole network level, some work (Leavitt 1950, Provan and Milward 1995) finds that highly centralized structures (i.e., whole networks in which a few nodes are highly central while most nodes have relatively low centrality) perform best. Note that the key theoretical mechanisms here is outflow — scholars concluded that high centralization was beneficial for whole network performance because the most central node played a crucial role in monitoring, dissemination, and leadership activities. Consider, also, that for a whole network to have high centralization, few members can pursue the "advantageous" central position —if all members sought to be highly central, the whole network would not be centralized. This highlights a tension — what is beneficial for a node at the ego level may harmful, en masse, for a whole network. Thus, we argue that there is a need for a sui generis theory and conceptual vocabulary at the level of the whole network.

We complement our theoretical development with an empirical application of the morphology framework, specifically to the performance of 250 interorganizational whole networks in the United States healthcare industry. These whole networks, known as accountable care organizations (ACOs), were formed as part of the Affordable Care Act with the goal of improving healthcare for specific patient populations.

Our paper makes several contributions. First, we introduce a novel framework and conceptual vocabulary for the study of whole network performance. The core argument of the morphology framework is that the relationship between whole network structure and performance is fundamentally contingent on other whole network properties. To be sure, our framework is not the first to recognize that the consequences of network structure may be contingent upon other (e.g., relational) factors (c.f., Nahapiet and Ghoshal 1998, Rowley et al. 2000, Ahuja et al. 2012). However, prior theory on the contingencies of structure has been designed for understanding ego networks. Thus, the framework we propose makes a contribution by leveraging different theoretical foundations from prior work (i.e., macrostructural sociology) to build a platform specifically for 
theorizing the contingencies of structure at the whole network level. Second, we draw on the framework to help reconcile conflicting findings on the relationship between structure and performance in the emerging whole networks literature. Rather than focusing on task moderatorswhich have not proven a robust strategy for resolving conflicting findings—we theorize that the structure-performance relationship must be understood by recognizing that structure does not exist in isolation, but rather coexists with two other-relational, cultural—whole network dimensions. Third, our study makes a substantive contribution by taking a whole network approach to new forms of organizing in healthcare delivery, an area in which outcomes above the organizational (i.e., at the whole network) level are crucial.

\section{THEORY DEVELOPMENT}

\section{Structure in Context: Insights from Macrostructural Sociology}

Efforts to understand whole network performance can be traced to the 1950s, when researchers used laboratory experiments to determine whether some whole network structures were better than others for group problem solving (Bavelas 1950, Guetzkow and Simon 1955). For instance, one study gave network members colored marbles. The experimenters then tasked groups with identifying the color that all members had in common (Macy et al. 1953). Subjects were allowed

to communicate by passing notes, but the researchers would modify the structural connectedness of the whole network-i.e. the extent to which network members could directly or indirectly reach one another. That is, experimental whole network configurations resembling stars or lines would constrain with whom notes could be shared. This literature provided early evidence that certain whole network structures may be superior to others.

Recent attempts to address conflicting findings on whole network performance harken back to these studies. Both classic and contemporary research has focused on the task being undertaken by network members to elucidate how structure may shape collective outcomes (Bavelas 1950, Mulder 1960). For example, Shore and colleagues (2015) found that highly connected whole networks excelled at finding diverse information; however, when required to use diverse information, such configurations tended to converge on fewer (potentially valuable) interpretations. Other studies 
have tried to disentangle these effects by altering the complexity of the problem or the options (e.g., explore, imitate) available to individual decision makers (Lazer and Freidman 2007, Mason et al. 2008, Barkoczi and Galesic 2016, Shirado and Christakis 2017).

Alternatively, evidence from the R\&D literature suggests that differently configured whole networks pursuing the same task—innovation—may produce similar outcomes. For example, increased innovation output has been associated with connected structures (Fleming et al. 2007), disconnected structures (Guler and Nerkar 2012), or both, contingent upon environmental factors (Funk 2014). Similarly, for problem-solving tasks, some research suggests that disconnected structures enhance performance by generating more novel solutions (Derex and Boyd 2016), while other studies find that more connected structures enhance performance because they enable members to better reconcile misaligned incentives (McCubbins et al. 2009), and facilitate more efficient error detection and imitation (Mason and Watts 2012).

Within this context, we propose an alternative approach to understanding the whole network structure-performance relationship. Our theory centers on the idea that structure is an incomplete representation of network activity. Structure is not the only driver of whole network outcomes because structure itself is inextricably tied to other (emergent) properties of the units that comprise it - nodes and ties. Our framework is grounded in the work of Blau (1964), who argued for the emergence of macro social structures ("macrostructure")—stratifications of society into different groups that share common features_-from patterns of recurring interactions ("microstructure"). Macrostructures "have emergent properties [e.g., enduring institutions] which are independent of the properties of individuals...in the sense that no individual can have equivalent properties" (Blau and Schwartz, 1997: x), but are themselves derived "from the simpler processes that pervade the daily intercourse among individuals" (Blau, 1964: 2). The primary driver for this aggregation was theorized by Blau to be the value that actors derive from social exchange and approval.

Although Blau argues that dyadic interactions are fundamental to more complex social structures, even dyads are, "of course, strongly influenced by the social context in which [they occur]. [Consequently] the analysis of social interaction in dyads, therefore, must not treat these pairs as if 
they existed in isolation from other social relations" (1964: 31). Here, "context" encompasses the broader network of relationships outside the dyad, both in the presence of the ties and in the nature or character of those ties, which may in turn be dependent on other factors, like specialization, power, or trust. Put differently, even a single connection between two individuals should not be viewed as a constant, but rather as a totem whose meaning or function is imbued by other dimensions of the interaction. This view of the plasticity of a relationship is similar to biological morphology, in which similar structures can perform differently precisely because they exist in different “contexts," such as an organism's size, taxonomic classification, or natural habitat.

Thus, at a baseline, the nature of interpersonal relations is important for understanding more complex, emergent group structure. To illustrate, Blau suggests that a higher-level construct like group cohesion emerges from individuals' collective efforts to gain approval from others (Blau 1964). Yet, without knowledge of this lower-level property of social interactions or the norms and values that support and propagate this behavior, one might believe that cohesiveness is a byproduct of the structure of relationships. Instead, Blau's insight is that the structure of relationships reflects only one dimension of cohesiveness; what looks to be well-connected group from the outside may be one of conflict and tumult.

As lower-level interactions coalesce into larger groups, a dilemma arises in Blau's efforts to link dyadic relationships with social structure. Because "there is no direct social interaction among most members of a larger community or entire society, some other mechanism must mediate the structure of social relations among them" (1964: 253). Here, Blau suggests that norms and values serve this mediating role. As social groups become larger, direct social interaction cannot be the only mechanism to maintain the greater social structure. "The legitimation of patterns of social conduct and social relations requires that common values and norms...reinforce and perpetuate them" (1964: 220). This set of norms and values, which Blau referred to as a group's culture or subculture, compensates for the lack of stabilizing qualities intrinsic to each interaction (e.g., interdependence) by providing guidelines for the behaviors expected and valued by the collective. Contemporary macrostructural scholars have embraced this interdependence in theories of social networks, 
demonstrating that structure emerges from the confluence of individuals and prevailing norms and values, and that, reflexively, the outcomes of structure are contingent on these factors (Centola 2015).

\section{Whole Network Morphology}

As noted earlier, we borrow from macrostructural theory the notion that the effects of whole network structure may be contingent on two other fundamental dimensions of social interaction—relations and culture-which, collectively, we refer to as the whole network morphology framework. In what follows, we organize our discussion of the interactions between the structural dimension and the relational and cultural dimensions around Table 1, which provides an overview of each.

\section{[Table 1 here]}

Structural dimension. First, the structural dimension (Table 1, first row) describes the structure of a whole network in terms of how its constituent relationships are arranged. Just as similar biological structures may appear in different organisms, similar whole network structures may be observed in whole networks that differ in composition of members, the types of relationships, or environmental context. Research on whole network effectiveness has focused on the structural dimension. For example, classic experiments in social psychology cited above utilized fixed structural configurations that varied in centralization (i.e., variance in network members' numbers of connections) (e.g., Guetzkow and Simon 1955). Other studies have focused on measures including density (e.g., Provan and Sebastian 1998), cohesion (e.g., Moody and White 2003, Buhr and OwenSmith 2010) and connectedness (e.g., Fang et al. 2010).

Relational dimension. The relational dimension (Table 1, second row) captures the nature or character of ties within a whole network, representing qualities like strength or duration. Studies of alliance (Dyer and Nobeoka 2000) and team (Reagans et al. 2005) performance often find that stronger ties benefit performance through enabling group members to work together more efficiently.

Past evidence hints that there may be an interaction between the structural and relational dimensions. Studying regional whole networks of law and venture capital firms, Buhr and Owen- 
Smith (2010) find evidence that in addition to strong ties, greater connectedness in institutionspanning whole networks has a positive relationship with performance. Dyer and Nobeoka (2000) similarly noted that Toyota's effective alliance network was characterized by many strong ties among members, but also a high level of connectedness. McFadyen and colleagues (2009) found that tie strength and density may interact negatively in the context of biomedical invention, where access to diverse information is important for performance. Rowley and colleagues (2000) found that strong ties in very dense networks led to worse firm performance. While these studies provide evidence that relational characteristics, such as tie strength, may influence ego level performance of a particular network position, macrostructural theory suggests that whole network performance is inherently dependent on the interaction between structural and relational dimensions.

The theoretical underpinnings of this interaction can also be found in Simmel's (1950) observations that the nature of dyadic relationships change in the presence of a third actor and in theories of structural balance (Davis 1967), where consistency in the nature (strength) of one's relationships with others is viewed as a determinant of observed structures. In the well-known "forbidden triad" (Granovetter 1973), if A is connected to both B and C, then the likelihood of a relationship between B and C forming is dependent on the strength of A's relationships with B and C. The stronger the A-B and A-C ties, the more likely B and C are to be connected. Although these simpler theories of dyadic interactions may be better suited to studying ego networks, they highlight how variations in the relational dimension (e.g., the strength of ties) can lead even a simple structure (triads) to function differently. Integrating these ideas, a whole network's relational dimension becomes a crucial lens through which to interpret its structural dimension.

Cultural dimension. Lastly, whereas the relational dimension focuses on the nature or strength of connections among whole network members, the cultural dimension (Table 1, third row) is concerned with the norms or values prevalent in the whole network (Blau 1964). Building on Durkheim's insights from Suicide that macrosocial forces (e.g., social and economic change) could influence individual behavior, Blau theorized that the values or orientations held by a majority could be important determinants of how a group, overall, behaved. He asserted that the confluence of 
shared values could also become an identifying trait of a group and influence members to act in accordance with the group's norms, even if it went against their individual orientations (Blau 1960). Thus, the cultural dimension describes a defining quality of the group that is influenced by members but is also distinct from the identity of any one individual.

Although the cultural dimension cannot be displayed using standard visualization techniques for whole network graphs, it is no less important. As Blau (1964) suggests, the cultural dimension becomes important as groups grow; the whole can no longer be stabilized by direct interactions, and the context within which relationships take place is essential to maintaining — and expanding — the social structure. Regarding whole network performance, the cultural dimension may therefore influence the types of behaviors that are valued within the group. For example, a culture that rewards competition may incentivize members to think more locally, in contrast to one which celebrates individual sacrifice for the greater good.

We distinguish the cultural dimension from the external institutional context within which a whole network may exist (Vasudeva et al. 2013, McFarland and Moody 2014). In defining the cultural dimension as an emergent characteristic of whole networks, we consider those external forces to represent the group culture insofar as members espouse them within the boundaries of the network.

Put differently, the whole network's cultural dimension may differ from the prevailing culture in the external environment if network members promote and propagate an alternate value system. In this way, the identities of the members are crucial ingredients that go into creating the cultural dimension of each whole network, and different whole network cultures may enact similar structures in various ways. Similar ideas have been noted previously in interpersonal networks (Brass et al. 2004), but macrostructural theory provides a foundation to extend to this observation broadly to whole networks, which may even (and often do) span organizational boundaries.

\section{EMPIRICAL APPLICATION: WHOLE NETWORKS IN HEALTHCARE}

We evaluate the ability of the morphology framework to generate novel predictions on the relationship between whole network structure and performance in a specific empirical context. We focus on the performance of a particular kind of whole network in healthcare-Accountable Care 
Organizations (ACOs).

Americans who are 65 years of age or older are eligible for government-funded insurance, provided by Medicare. Since its creation in 1965, Medicare has become the country's largest insurer, covering more than 50 million people. Due to older patients' more complex health care needs, multiple types of providers—spread across organizations and health systems—participate in the care of Medicare beneficiaries. This complexity requires collaboration to make sure the right care is provided at the right place and time. However, such collaboration is de-emphasized by the system, which rewards providers primarily for the quantity of work performed, leading to overuse and high costs. Consequently, reining in spending is a central concern for Medicare's administrator Centers for Medicare and Medicaid Services (CMS).

Provisions of the 2010 Affordable Care Act gave rise to the Medicare Shared Savings Program ACOs, a large-scale effort to address fragmentation within the healthcare system. Through contracts between CMS and groups of healthcare providers, ACOs receive financial incentives to promote coordination and prevent what Berwick (2002) described as healthcare's fatal flaw: "every discipline for itself, every guild for itself ... [the assumption] that either we will preserve quality or cut costs." Each ACO is given an annual performance benchmark based on historical expenditures for a particular population of at least 5,000 beneficiaries (CMS 2017). ACOs can reap up to $50 \%$ of the savings they generate if they reduce their spending below their benchmarks by at least $3 \%$. Thus, cost-savings is the primary performance outcome for ACOs. There is no direct incentive for improving quality — in fact, ACOs were not even penalized for poor quality in the first year (2013).

Although ACOs have a formal contractual basis, experts see them as "a peculiarly social intervention, using social levers — shared accountability and collective incentives - to encourage new relationships among groups" (Kreindler et al. 2012). Thus, attainment of their economic goals is expected to be dependent on ACO members' ability to collaborate.

\section{HYPOTHESES}

Before moving forward, we first lay some additional theoretical groundwork to connect the whole network morphology framework to our empirical context. ACOs are "goal-directed" whole 
networks (Provan and Kenis 2008) in which performance depends on collaboration. Therefore, our hypothesis development proceeds under the assumption that variations in network morphology will affect the manner in which members of a network interact with one another. Secondly, although the structural dimension of whole network morphology can be characterized according to several properties, we focus on connectedness - i.e., the extent to which whole network members are mutually reachable through direct or indirect relationships (Lazer and Friedman 2007). As discussed previously, connectedness has important implications for the ability of whole network members to collaborate, and received much attention in whole network research (Kearns et al. 2006, McCubbins et al. 2009, Fang et al. 2010, Mason and Watts 2012, Shore et al. 2015, Derex and Boyd 2016). In what follows, we consider whether interactions between the structural dimension of whole network morphology on the one hand and the relational and cultural dimensions on the other may moderate how structural connectedness affects whole network functioning and outcomes.

\section{Structural Dimension $\times$ Relational Dimension}

First, we consider how the relational dimension of whole network morphology may moderate the structure-performance association in ACOs. Like the structural dimension, the relational dimension encompasses multiple whole network properties. Below, we focus on relational strength, understood as the overall frequency of interactions within a network (Reagans et al. 2005), which has been important in health care research (Everson et al. 2017).

Greater connectedness is likely to provide benefits through better information flow, but may also increase information processing demands on network members. Increasing relational strength in a highly connected whole network may ameliorate some of these demands through mutual learning and more efficient knowledge transfer (Uzzi 1997, Hansen 1999, Zollo et al. 2002, Levin and Cross 2004, Reagans et al. 2005).

Whole network performance may also be enhanced by greater connectedness if there are benefits for cooperation (Okhuysen and Bechky 2009, Gulati et al. 2012). However, as connectedness increases, so does the burden of managing a large number of relationships, or relational complexity. Considering this trade-off, the benefits of greater relational strength may 
similarly tip the scales towards better cooperation by making it easier to avoid or resolve conflicts, and enhance trust within the collective (Zaheer et al. 1998).

However, under conditions of low connectedness (i.e., greater disconnectedness), the implications of greater relational strength may change. Lower connectedness makes it more difficult for network members to share information and reduces opportunities for members, on the whole, to interact with others in the group. Therefore, despite being part of the same whole network, more members are at greater risk of feeling detached and may further not experience the same pressures to contribute to the collective. In fact, increasing relational strength in more isolated positions may push network members towards parochial, or highly localized, orientations because members on average only have a few direct connections. In other words, even though some members are formally part of a larger group, they may also feel attachment to only a comparatively small number of partners (Lau and Murnighan 1998, Heidl et al. 2014, Hollingsworth et al. 2015a; Ghomrawi et al. 2018). Consequently, as connectedness decreases, greater relational strength may actually harm whole network performance by reinforcing "siloed" positions. Thus, we propose a first hypothesis. Hypothesis 1 (H1): As structural connectedness (disconnectedness) increases, greater strength in the relational dimension of whole network morphology will be associated with better (worse) ACO cost performance.

\section{Structural Dimension $\times$ Cultural Dimension}

Turning to the cultural dimension, we first describe a common culture in health care that emphasizes the professional autonomy of physicians. ${ }^{3}$ Subsequently, we consider how the presence of this cultural orientation in ACOs may alter the whole network structure-performance relationship.

In the United States, the medical profession has historically exercised great power over health care delivery (Starr 2008, Stevens 1998); consequently, the culture of health care delivery has tended to reflect physician values. Among those values, perhaps none has been as important as autonomy (Freidson 1988), what Pont (2000: 99) has called, "the highest ethic of the medical profession." Professional autonomy refers to the ability for an individual to practice his or her work

\footnotetext{
3 We use the term "autonomy" specifically in reference to theories of professional autonomy (e.g., Abbott 1988, Light \& Levine, 1988). Thus, our use of the term autonomy is different from the network structural conception of autonomy (i.e., low network constraint [Burt, 1992]).
} 
according to his or her discretion— to exercise local control. For physicians, autonomy manifests in freedom over patient therapies, whether and when to refer patients for consultations, and with respect to organizing and managing patient records. Underscoring this culture of physician autonomy, in the United States, decisions made by physicians are often treated with deference, particularly relative to other professionals. Similarly, although physicians are the most powerful professional figures in hospitals, in many health systems they are not hospital employees, but rather are contractors with freedom to work at multiple institutions.

Cultures of health care delivery are not, however, universally oriented towards physicians. Historical (Stevens 1998) and sociological (Freidson 1994, Ritzer and Walczak 1988, Starr 2008) accounts have documented a decline in physician autonomy over time. Reinforcing this trend, reform advocates have become vocal in pushing away from the physician-centric model (e.g., Rickert 2012, Okie 2012). Many observers note that organizational values (e.g., financial performance) have become prevalent, a trend attributed to the corporatization of medicine (Starr 2008). The growth of professional groups like nurses and pharmacists has also been accompanied by an emerging cultural orientation towards interprofessional collaboration (Horn and Jacobi 2006). Finally, with growing concerns about cost and quality, population health and patient-centered care have become important culture orientations (Berwick et al. 2008). Today, health care delivery encompasses a multiplicity of cultural orientations, alongside physician autonomy.

Research suggests that different cultural orientations are likely to have implications for physician behavior. These implications become apparent when examining the tensions between different orientations. Consider the tradition of physician autonomy alongside more the recent orientation towards organizational values. Particularly in the latter, due to the proliferation of expensive drugs and treatments, physicians are almost always restricted in what they may offer patients. For example, although surgeons may have preferences regarding the brands of implants they offer patients, hospitals' cost containment measures (e.g., efforts to achieve economies of scale in negotiations with distributors) often limit acting on these preferences.

Similar to our discussion of the interaction between whole network structure and the 
relational dimension, we do not consider the physician cultural orientation to be, on average, better or worse for collective performance. Instead, based on the morphological framework, our expectation is that the performance implications of a whole network culture are best understood when taken together with the structural dimension.

To begin, greater whole network connectedness likely increases the information processing demands on whole network members because they are receiving and sending information—such as patient records and treatment plans in the case of ACOs-to a greater multiplicity of partners. However, a shared orientation towards physicians' professional autonomy may afford network members greater freedom or discretion to determine on what to focus. For similar reasons, an emphasis on physician autonomy may help network members feel more comfortable declining requests, which tend to increase with connectedness (Portes and Sensenbrenner 1993). Similarly, considering the positive relationship between connectedness and relational complexity, a physicianoriented culture may also create greater allowances for network members to exercise different views and practices (Kelly and Kelly 1994), thereby mitigating conflict. For example, a group of diverse specialists may be highly connected but avoid conflict due to a shared understanding that each partner is expert at what they contribute to the collective.

When a whole network structure becomes less connected, however, a culture of physician autonomy may have negative implications for the structure-performance relationship. Physician autonomy may exacerbate the challenge of network members being fragmented into smaller, localized groups because the autonomy orientation further encourages physicians to behave independently. Thus, paralleling our arguments on high relational strength within disconnected whole networks, an orientation towards physician autonomy may promote heterogeneous views and siloing among whole network members, limiting the ability of the whole to cooperate and achieve a shared goal. To use the same example of a group of diverse specialists, partners that lack any interaction may be less inclined to trust the capabilities or commitment (to the whole) of those with whom they have no contact. Consequently, cooperation may be achieved in connected subgroups but may fail to extend across structural faultlines (Heidl et al. 2014). 
In sum, a culture of physician autonomy likely attenuates the information processing demands and relational complexity associated with connectedness, while accentuating the challenges of decreasing connectedness for shared perspective. Thus, we propose a second hypothesis.

Hypothesis 2 (H2): As structural connectedness (disconnectedness) increases, the presence of an orientation towards physician autonomy in the cultural dimension of whole network morphology will be associated with better (worse) ACO cost performance.

Figure 2 illustrates our hypotheses by visualizing the morphology of two ACOs. These ACOs are similar in some respects. Both are relatively large, with ACO A comprising 41 organizations and ACO B 75 (the mean is 26). Both are based in large metropolitan areas. However, morphologically, ACOs A and B are different. ACO A has greater connectedness than ACO B, which has multiple sparsely connected clusters and several isolates. Relative to ACO B, ACO A also has greater relational strength, as evidence by a greater number of thicker ties. Finally, differences in cultural orientation are shown using the yellow and blue backgrounds for the presence and absence of culture of physician autonomy in ACOs A and B, respectively.

[Figure 2 here]

\section{DATA AND METHODS}

The first MSSP ACOs began on April 1, 2012, with additional starts on July 1, 2012 and January 1, 2013. Subsequently, new ACOs joined the program on the first of each year. We focus on the 392 ACOs founded between April 2012 and January 2015 and track their performance from 2013 - the first performance year — through 2016 — the last year available at the time of data collection. After dropping observations from Puerto Rico and cases with missing data, our sample includes 250 ACOs for which we have at least two years of complete records.

\section{Mapping Whole Network Structure in Healthcare}

\section{Beneficiary sharing relationships: Conceptual and contextual backgtound. Our}

analysis focuses on the whole network structure-performance relationship in ACOs. To examine this association, we first need to identify and measure relevant relationships (i.e., ties) among ACOs' organizational members. Our theoretical development suggests that these relationships should meet several requirements. First, they should be consistent in nature so that whole network properties can 
be interpreted similarly for all ACOs in our sample. Second, they should be linked to shared work (requiring coordination and cooperation) among ACO member organizations. Third, considering our focus on whole network performance, shared work should represent activities that bear on an important collective outcome. Since our endpoint is the ability of ACOs to reduce spending, the relationships should be relevant for understanding costs.

Beneficiary-sharing relationships meet these requirements and have additional characteristics that make them attractive. ${ }^{4}$ Beneficiary-sharing relationships have been used extensively in health services research as a proxy for physician-to-physician interactions (Barnett et al. 2011, Landon et al. 2012, 2013, Hollingsworth et al. 2015b). Beneficiary sharing occurs when healthcare providers treat and subsequently file an insurance claim for the same patient. Unsurprisingly, one of the most common reasons for beneficiary-sharing is patient referral (i.e., when a physician instructs the patient to consult another doctor). However, beneficiary-sharing is also initiated by patients (i.e., when an individual seeks care from multiple providers) (Bourgeois et al. 2010). In both instances, care requirements generally demand that patients' medical records be communicated from one provider to the next (e.g., Vogus et al. 2010). Due to these communication needs, beneficiary-sharing ties often coincide with professional relationships among physicians. ${ }^{5}$

Primary care offers a useful illustration of how beneficiary-sharing relationships form. Primary care providers (PCPs) are typically the first point of contact between a patient and the healthcare system (Friedberg et al. 2010). When a beneficiary has complex needs, a PCP may refer him or her to a specialist physician for treatment (Wagner et al. 1996). The beneficiary may then follow up with the referring PCP. As this process repeats with other beneficiaries, the PCP and specialist are likely to develop a deeper relationship.

Beneficiary-sharing relationships are also valuable for data considerations. For administrative

4 "Beneficiary" refers to patients who are covered under a particular insurance plan, such as Medicare.

5 As we discuss in greater detail below, recent work finds that interpersonal relationships among physicians continue to be important even as the use of electronic medical records has become more common. In particular, electronic health records are not yet ubiquitous (Cross et al. 2015), nor are they necessarily transferrable if organizations are using different systems (e.g., Siemens versus Epic). Moreover, electronic medical records are limited in their ability to transfer complex or tacit knowledge, which remains important in medical care. 
purposes, the provision of health care services creates robust records of patient-physician and patient-organization interactions (Barnett et al. 2011). In order for a physician or organization to be paid for services, a claim for reimbursement must be submitted to the patient's insurance company (Beck and Margolin 2010). As these claims are submitted and processed, they create administrative "breadcrumbs" that trace a beneficiary's journey among providers. Moreover, because reimbursements are on the line, these data represent a very complete record of work that is done for Medicare beneficiaries, which represent some $98 \%$ of all Americans over age 65 and $99 \%$ of all deaths within that age group (Funk et al. 2017).

Using multiple methods, studies have validated the use of beneficiary-sharing relationships as proxies for physician networks. Most convincingly, research has shown that shared beneficiaries correlate with physicians' self-reported professional relationships. Barnett and colleagues (2011) surveyed 616 physicians about their referral and advice-seeking relationships with other geographically proximate physicians. The authors compared these survey results to patterns of shared beneficiaries observed in Medicare claims. They found that if the threshold for recording a tie based on Medicare claims was set to nine or more common beneficiaries (over a year), there was an $82 \%$ overlap with ties found using surveys.

Another study (Landon et al. 2013) used community-detection algorithms on beneficiarysharing patterns to inductively determine meaningful groups of physicians serving 4.5 million Medicare beneficiaries. The authors found that this approach — a purely network analytic methodmapped more meaningfully onto patient care patterns than groups based on the hospital to which each physician made the most referrals. Thus, defining regional healthcare systems as the constellation of physicians around hospitals was less predictive of actual patterns of patient-physician visits than clusters that emerged from more complex beneficiary-sharing networks (i.e., that included direct and indirect relationships). Such research provides strong support that beneficiary-sharing ties are a good proxy for physician relationships.

Finally, studies have helped to validate the use of beneficiary-sharing relationships as proxies by examining cost and quality outcomes, finding strong associations between network properties and 
important endpoints in areas where social factors would be expected to matter, but not where more technical or infrastructural factors would likely predominate (Funk et al. 2017).

Beneficiary sharing relationships: Data and measurement. To map whole beneficiarysharing networks among ACO member organizations, we began with physician-level data derived from Medicare claims, made publicly available through a Freedom of Information Act request. ${ }^{6}$ These data record the number of shared beneficiaries between all pairs of Medicare physicians, identified by unique National Provider Identifier (NPI) numbers. Physician pairs enter the data if, within a 60-day window, both physicians bill for services provided to the same Medicare beneficiary. Due to privacy regulations, dyads are only reported for providers who shared at least 11 beneficiaries over a calendar year. For our study, however, this restriction is fortuitous, because as described above, physician dyads with fewer shared beneficiaries less likely reflect meaningful relationships (Barnett et al. 2011).

For each year in our study, the full network data capture more than 70 million beneficiarysharing relationships. From this universe of ties, we use Medicare Research Identifiable Files (RIFs) — obtained through a request to CMS — to isolate participating physicians and organizations in each ACO, in each year of the MSSP program. ${ }^{7}$ Using physicians' organizational affiliations within ACOs, we aggregate physician-level ties to the interorganizational level using a three-year window. Our use of a rolling window is consistent with prior research on interorganizational networks (e.g., Gulati et al. 2012). More importantly, our approach aligns with CMS' methodology for assigning patients to ACOs and determining benchmarks, which are based on patients treated by ACO members, and the costs incurred, during the prior three years (CMS 2017).

Our final sample of 250 ACOs comprises approximately 250,000 physicians working in 44,231 organizations. More information on network construction is in the Supplementary Appendix.

\section{Dependent Variable}

Cost performance. Our outcome is the extent to which an ACO achieves savings relative to

\footnotetext{
${ }^{6}$ CMS provides more detailed information on the beneficiary-sharing (referral) data (accessed Feb 28, 2018): https:/ / questions.cms.gov/faq.php? faqId $=7977$.

7 The key files for this step are the Medicare Shared Savings Program ACO Provider RIFs.
} 
the CMS benchmark. We compute this variable by calculating the ratio of total savings (or losses) to the benchmark, multiplied by 100 , for each ACO $\times$ year observation. Because losses are also captured, performance can be negative. Our outcome is the same measure CMS uses to evaluate ACO performance and therefore is the metric ACOs are incentivized to maximize. If ACOs fail to save at least 3\% of their benchmark, they receive no incentive payments regardless of quality. Therefore, while quality is important—incentive payments may be reduced for low quality—it is not the primary ACO performance metric. We measure performance at $t+1$; other variables are measured at $t$ (c.f., Reagans et al. 2005).

\section{Independent Variables}

Structural disconnectedness $\left(\boldsymbol{S}^{D}\right)$. To evaluate whole network structure-performance relationship in ACOs, we operationalize the structural dimension of whole network morphology as structural connectedness. We capture structural connectedness using a measure developed by Borgatti (2006) to quantify fragmentation in whole network structures. Thus, our proxy for whole network structure is a measure of disconnectedness; lower values correspond to more connected networks. Given our purposes, this measure, which we refer to as $S^{D}$, is attractive because it integrates, into a single index, several different aspects of whole network connectedness, including the number of components (i.e., sets of nodes that are mutually reachable through direct or indirect ties) within the network, the relative size of each component, and the relative connectivity (i.e., path length) within each component. Formally, $S^{D}$ is defined as

$$
S^{D}=1-\frac{2 \sum_{i>j} \frac{1}{d_{i j}}}{n(n-1)}
$$

where $\frac{1}{d_{i j}}$ represents the connectivity between pairs of nodes based on shortest paths. $S^{D}$ ranges from 0 to 1 ; the measure takes on a maximum of 1 when the whole network structure consists of only isolates and a minimum value of 0 when the network is completely interconnected.

Relational strength $\left(\boldsymbol{R}^{\boldsymbol{s}}\right)$. Within the ACO context, we used our morphology framework to predict that greater strength in the relational dimension will be associated with worse performance as structural connectedness increases (Hypothesis 1). To evaluate this prediction, we operationalize 
relational strength as the average number of shared beneficiaries in each ACO (Berman et al. 2002). ${ }^{8}$

Formally,

$$
R^{S}=\frac{\sum_{e} w_{e}}{n_{c}}
$$

where $w_{e}$ is the weight (i.e., shared beneficiaries) of edge $e$ in the network and $n_{c}$ is the number of connected (non-isolate) nodes. To facilitate interpretation, we divide this value by 10,000.

\section{Cultural orientation (Co).}

Finally, the framework we propose suggests that the structure-performance relationship may be altered by the cultural dimension of whole network morphology. In ACOs, we predicted that this relationship would differ systematically depending on the presence or absence of a physician cultural orientation (Hypothesis 2). Investigating this hypothesis poses an empirical challenge-although prior work has discussed a role for cultural orientation in whole networks (Provan and Milward 1995, Nahopiet and Ghoshal 1998, Owen-Smith and Powell 2007), and specifically within ACOs (e.g., Kreindler et al. 2014), no definitive taxonomy of cultural markers has been established.

To address this challenge, we turned to research on organizational culture and identity. While culture and identity are not equivalent, previous work has shown a link between the two (Hatch and Schultz 2002), typically viewing identity as a manifestation of culture, "to which individuals can commit their emotions and energies" (Pettigrew, 1979: 578). Drawing on anthropology, organizational scholars have also noted that organizations create cultural symbols that “evoke emotions and impel men to action" (Cohen, 1974: 23). In that vein, identity is something that is actively constructed, particularly in the gestational stages of organizational development (King et al. 2011). Extending this to the interorganizational context, other research has pointed to the importance of shared or collective identity as a stabilizing force in collaborations and alliances (King et al. 2010), underscoring the significance that identity construction likely also has for formal, goaldirected whole networks, such as ACOs.

One particularly salient symbol of organizational identity that provides a window into culture and has

\footnotetext{
${ }^{8}$ Reagans and colleagues (2005) define an alternative measure that adjusts for possible ties. Although attractive, this adjustment is inappropriate for our study because it would introduce structure into the measure of relational strength.
} 
"significant functional consequences" (Pettigrew, 1979: 574) is the name. Scholars have theorized that organization names "encode central features of meaning and organizational identity" (Glynn and Abzug, 2002: 267). Researchers also note that a name is one of the first things that confers existential meaning to individuals, organizations, or even social movements (Cerulo 1997). For example, the name chosen by the founders of an organization not only makes a fledging collective something "real," but also helps communicate cultural values to members. To the extent that names help communicate organizational values, they are likely an important conduit through which individuals relate to an organization as it grows. This identification is a key part of socialization and is thought to be necessary for communication among members, who may otherwise have little in common as a basis for interaction (Kogut and Zander 1996. Indeed, recent work has revealed that the "language that [organization members] use to describe the organization as a whole" can be used to systematically examine differences in organizational culture across a large sample (Corritore et al. 2019), as well as to track how both new and existing members acculturate to the prevalent cultural norms (Srivastava and Goldberg 2017; Srivastava et al. 2018).

Given the newness of the ACO program, the name chosen by particular ACOs may have important effects on the way members perceive what the ACO is and what it stands for, priming members to behave in different ways (Oyserman and Lee 2008) and effectively informing part of their cultural tool kit (Swidler 1986). Empirically, we observe wide variation in ACO names. Some names are simply administrative sounding acronyms, such as "AzPCP-ACO, a Medical Corporation" or "APCN-ACO, a Medical Professional Corporation." Other ACOs clearly derive their name from and thus identify with a large, central member, like the "Lahey Clinical Performance ACO" or “Cedars-Sinai Accountable Care." Such ACOs also exhibit other traits that suggest a clear organizational anchoring (e.g., their websites are a subsidiary page of the central organization, rather than a unique domain).

Many ACO names, however, show greater distinctiveness, often through language that relates to values, like the central role of physicians. For example, "Georgia Physicians for Accountable Care" evokes the image of a group of physicians intent on providing accountable care 
for their patients. Consistent with this interpretation, the ACO describes on its website (with an independent domain name) that "[its] goal is to make a fundamental change in the way healthcare is delivered through ensuring high quality and effective care while enhancing the patient experience" (www.gpaco.org 2019). The name "Independent Physicians' ACO of Chicago" evokes similar values. The website highlights that the ACO is " $100 \%$ physician owned... formed entirely by practicing physicians" and "aims to increase the quality of care while reducing overall health expenditures for patients" (www.aco-chicago.com 2019).

While CMS requires that every $\mathrm{ACO}$ has a name, it does not dictate naming requirements (e.g., needing to contain terms like "accountable care"). Thus, naming choices are likely by internal considerations, presumably in the service of crafting or revising a group identity, both for members and patients. Consequently, we argue the name selected by each ACO is a reasonable indicator of its cultural orientation.

Methodologically, we follow a large tradition of prior research that leverages names, labels, and other textual data to identify properties of culture and identity (Glynn and Abzug, 2002; Pachuki and Breiger, 2010; King, 2011; Granqvist et al., 2013; Bail, 2014; Kozlowski et al., 2014; Grossman and Varnum, 2015; Doyle et al. 2017; Srivastava and Goldberg 2017; Srivastava et al. 2018; Corritore et al. 2019; Jackson et al., 2019). Specifically, our primary approach for analyzing ACO names was to code the terms of their constituent text. Guided by our theory development, we focused on determining whether a given name indicated a physician cultural orientation. We began by cleaning the names (based on MSSP ACO Participant files) of all ACOs active between 2012-2016. We removed punctuation, collapsed common terms (e.g., state names) into abbreviations, and stemmed words using Porter's (1980) algorithm (e.g., "collaborative," "collaboratives," and "collaboration" were converted to "collabor") Then, two of the authors categorized whether each of the resulting 697 unique terms evoked the image of physicians or the central role of physicians in patient care. The third author resolved any discrepancies between the two coders. Key terms included "physician," "doctor," "md," "med," "clinic," “practice," and "primary." Finally, for each ACO, we counted the physician-oriented terms to determine the cultural orientation. The terms "physician," "doctor," and 
"md" were considered to unambiguously indicate a physician cultural orientation, whereas the others were weighted against the presence of other culturally distinctive terms. Using this methodology, we created a variable for the cultural orientation of each $\mathrm{ACO}, \mathrm{C}^{\circ}$, where $\mathrm{C}^{\mathrm{O}}=1$ if the $\mathrm{ACO}$ has a physician cultural orientation. ${ }^{9}$

We evaluated the face validity our measure by systematically analyzing the full text of ACO websites. Specifically, we scraped the full text of the landing page of ACO websites (which typically contain self-descriptions and mission statements). Subsequently, we examined the frequencies with which physician versus nonphysician ACOs used different words. We based this approach on previous work in organizational identity, which analyzed the text of mission statements (King et al. 2011). We found strong evidence supporting the face validity of our hand-coded variable (see Appendix D and Figure S2). In particular, words like “independent," “doctors," "physicians,” and "experience" appeared much more frequently in the text of websites for ACOs with a physician orientation $\left(C^{O}=1\right)$. Among the top words associated with a nonphysician orientation $\left(C^{O}=0\right)$ were "corporate," "manage," "governance," and "regional," suggesting a more organizational or community-oriented focuses.

\section{Control Variables}

Patient and provider characteristics. We control for Total providers in each ACO, which includes PCPs, specialists, nurse practitioners (NPs), clinical nurse specialists (CNSs), and physician assistants (PAs). Primary care physicians are seen as critical for care coordination, while specialists provide a higher level of care and therefore are more expensive. NPs, CNSs, and PAs perform primary and specialty care roles and thus contribute to ACO capacity. We also control for Total beneficiaries assigned to each ACO.

Additionally, we account for the demographic composition of ACO beneficiaries by including variables for $\%$ Female beneficiaries, $\%$ Black \& Hispanic beneficiaries, \% Elderly beneficiaries (age 85+), \% Disabled beneficiaries, and \% End-stage renal disease (ESRD) beneficiaries. Each of these categories

\footnotetext{
${ }^{9}$ Additional methodological notes are in the Supplementary Appendix. Coded names are available on request.
} 
represents a patient population that is associated with increased spending (Lassman et al. 2014) or more complex health and socioeconomic statuses (Kaiser Family Foundation 2016). al.

General ACO characteristics. We adjust for characteristics of ACOs that are not directly related to patients or physicians. First, to differentiate ACOs that were provided with financial support from CMS, we include a dummy variable, ACO Advance Payment, set to 1 in years where an ACO received such support. Next, we control for each ACO's Quality score, measured at $t+1$. CMS reports a composite quality score (out of 100) for ACOs based on 33 metrics, covering patient/caregiver experience, care coordination/patient safety, preventative health, and factors related to at-risk populations (e.g., diabetes, heart disease) (CMS 2017). Quality is an important control because it helps account for latent variation in organizational capabilities (e.g., leadership, resources). We also include an indicator for $A C O$ spans noncontiguous states. While relatively uncommon, the morphology of ACOs with noncontiguous service areas may manifest differently due to greater geographic distance or different strategies for achieving Cost performance.

In addition to these factors, we also adjust for whole network characteristics of ACOs. First, we control for Network size, defined as the number of member organizations that comprise each ACO. Next, we control for Network isolates, defined as the number of organizations in each ACO with no interorganizational ties. Physicians in these organizations share beneficiaries internally, but not with physicians in other organizations. Additionally, we include a control for $\%$ Non-referring to account for the proportion of nodes within each ACO that do not share beneficiaries with other member organizations. The numerator of this variable includes self-referring Network isolates but also adds organizations that are not associated with any beneficiary sharing activity at all. These latter organizations account for a minority of ACO members $(\mathrm{N}=1,598 ; 11 \%$ in 2014); we describe these organizations in the Supplementary Appendix. Finally, we also control for Network turnover in each ACO, which reflects changes in organizational membership.

\section{Model Estimation}

Our dependent variable is continuous with a roughly normal distribution and we have annual observations for each ACO. Thus, we model Cost performance with fixed-effects OLS regressions of 
the form

$$
y_{i t+1}=\mu_{t}+x_{i t} \beta+c_{i}+\epsilon_{i t}
$$

These models assume that the performance of ACO $i$ at time $t+1$ is a function of four components. First, $\mu_{t}$, captures time-varying factors that affect all ACOs equally. Second $x_{i t}$ captures time-varying factors that differ for each ACO, including the independent variables, interactions, and controls. Third, $c_{i}$ captures all unobserved time invariant heterogeneity among ACOs. Finally, $\epsilon_{i t}$ is an idiosyncratic, time-varying error term for each ACO-year. As is common when interaction terms are included, the variance inflation factors (VIFs) are increased for Relational strength variable (VIF = 20.77) and its interaction with Structural disconnectedness (VIF $=18.35)$. The remaining VIFs are 10, with a mean VIF of 5.41.

\section{RESULTS}

Descriptive statistics and correlations for all variables can be found in Appendix E, Table S4. There are 736 whole network $\times$ year observations in our sample, comprising 250 ACOs.

[Table 2 here]

\section{Fixed-effects Regression Results}

Table 2 displays the fixed-effects regression results of Cost performance on Structural disconnectedness and the interactions of Structural disconnectedness with Relational strength and Cultural orientation. Robust standard errors are in parentheses. Independent variables (with the exception of Cultural orientation, which is binary) are mean-centered. Model 1 includes only controls. Model 2 includes only the variables for the structural, relational, and cultural dimensions of whole network morphology. Model 3 adds the controls. Model 4 introduces the interaction term between Structural disconnectedness and Relational strength. Model 5 shows the interaction between the Cultural orientation variable and structure. Model 6 shows only the key independent variables and interactions. Finally, Model 7 is the full model, to which we will primarily refer in our discussion.

Whole network structure and performance. Before turning to our hypotheses, we consider the relationship between whole network structure and performance. Recall that in our theory development, we drew on findings from prior work to observe that Structural disconnectedness may be 
either positively or negatively associated with Cost performance. Consistent with this view, we do not find robust evidence of an association between Structural disconnectedness and Cost performance (Table 2, Model 7: $\beta=-0.31, p=0.918)$.

Assessments of the hypotheses. We find, however, that when we introduce a moderator for Relational strength, the association between whole network structure and performance is clearer. In Model 7 of Table 2, the coefficient for the interaction between Structural disconnectedness and Relational strength is negative and statistically significant $(\beta=-0.30, p=0.005)$, offering support for H1's prediction that as structural connectedness decreases, greater Relational strength will be negatively associated with Cost performance. This result is consistent with the possibility that as relational strength goes up, subgroups of actors become more siloed, making it difficult for network members to collaborate in pursuit of collective goals.

Next, we turn to models in which the structure-performance relationship is examined under different cultural orientations. Using our hand-coded measure-where $C^{O}=1$ if the name of the ACO reflected physician autonomy, and 0 otherwise- the coefficient for the interaction between Structural disconnectedness and physician Cultural orientation (Table 2, Model 7: $\beta=-21.86, p=0.012$ ) suggests a different structure-performance relationship depending on the presence or absence of a physician Cultural orientation. Increasing Structural disconnectedness is negatively associated with whole network performance in the presence of a physician Cultural orientation. This finding supports H2's prediction that the Structural disconnectedness-Cost performance relationship would be negative in ACOs with a physician Cultural orientation.

[Figure 3 here]

\section{Interpreting the Regression Results}

Structural disconnectedness $\times$ Relational strength. The left panel of Figure 3 shows predicted values of Cost performance at different levels of Structural disconnectedness and Relational strength. The first set of bars on the left show Cost performance for ACOs with low Structural disconnectedness (10 ${ }^{\text {th }}$ percentile) at low (10 $0^{\text {th }}$ percentile), moderate (median), and high ( $90^{\text {th }}$ percentile) Relational strength. There is not a large difference in terms of expected Cost performance based on Relational strength; there is 
a slight negative trend as Relational strength increases, but the differences are not significant. However, at moderate or high levels of Structural disconnectedness, there is a more dramatic difference in Cost performance as Relational strength changes. When Relational strength is low, Cost performance does not change much with Structural disconnectedness (in fact, it increases slightly as disconnectedness goes up). At moderate Relational strength, however, Cost performance is significantly lower at moderate Structural disconnectedness than what it was at low Structural disconnectedness (0.9\% savings vs. $1.7 \%$ savings, respectively), and even worse at high Structural disconnectedness (0.3\%overspend). At high Relational strength, the difference is even greater, with negative Cost performance in ACOs at moderate and high levels of Structural disconnectedness (a $0.2 \%$ overspend for moderate Structural disconnectedness and $2.5 \%$ overspend for high Structural disconnectedness, compared to 1.6\% savings for low Structural disconnectedness).

These differences can be contextualized using the (2016) average ACO benchmark of $\$ 205$ million. At low Structural disconnectedness, an ACO with low Relational strength is expected to save 1.8\% (\$3.7 million). At high Structural disconnectedness and low Relational strength, an ACO is still be expected to generate $2.0 \%$ savings ( $\$ 4.1$ million). Now, if the ACO had high Relational strength, it is still expected to perform relatively well at low Structural disconnectedness_-1.6\% savings ( $\$ 3.3$ million). However, if both Structural disconnectedness and Relational strength are high, performance changes dramatically to a projected overspend of $2.5 \%$ (i.e., a $\$ 5.1$ million loss).

Structural disconnectedness $\times$ Cultural orientation. The right panel of Figure 3 plots predicted values for the interaction of Structural disconnectedness $\left(10^{\text {th }}, 50^{\text {th }}\right.$, and $90^{\text {th }}$ percentiles $)$ and Cultural orientation. Recall that we found support for our prediction that the whole network structureperformance relationship would be contingent on the presence or absence of a physician Cultural orientation. Figure 3 unpacks this relationship. Consistent with H2, when Structural disconnectedness is low, a physician Cultural orientation is associated with better performance. However, as Structural disconnectedness increases, a physician Cultural orientation is associated with worse performance, while performance without this orientation is more stable. At low Structural disconnectedness, physician Cultural orientation is associated with high Cost performance, a savings of $16.0 \%$, about $\$ 32.8$ million based on the average benchmark. In contrast, the absence of physician Cultural orientation is associated with savings 
of nearly zero, or $0.2 \%(\$ 410,000)$. This surprising result suggests that holding other factors constant, at low Structural disconnectedness, the performance differential associated with the presence or absence of a physician Cultural orientation could be more than $\$ 30$ million.

Now, consider how this relationship changes as Structural disconnectedness increases. At moderate Structural disconnectedness, the gap between the presence and absence of a physician Cultural orientation shrinks to about 7.9\%. At high Structural disconnectedness, physician Cultural orientation is associated with lower performance, with a predicted overspend of $2.4 \%$, whereas in the absence of a physician Cultural orientation performance is closer to neutral (-0.2\%), a differential of only $2.2 \%$ between the presence and absence of a physician Cultural orientation. This suggests that with a physician Cultural orientation, more connected whole network structures are associated with much better performance than more disconnected ones. However, in the absence of a physician Cultural orientation, the structure-performance relationship is relatively more stable.

\section{Additional Analyses}

To evaluate the robustness of our findings, we conducted several additional analyses, shown in the Supplementary Appendix (Table S4).

Alternative proxy for structural disconnectedness. We began by evaluating the robustness of our findings to alternative proxies for the whole network dimensions. As an alternative for the structural dimension, Model 8 measures disconnectedness using average path length (e.g., Lazer and Friedman 2007). The results are consistent with our main findings.

Alternative proxy for relational strength. We also considered an alternative measure of the relational dimension. Model 9 replicates our main model, but proxies for relational strength using average shared beneficiaries weighted by the total number of organizations, including isolates and disconnected organizations that are not associated with any beneficiary-sharing activity. Findings using this approach are consistent with our main results.

Alternative proxy for cultural orientation (machine learning). Model 10 replicates our main models using a machine-learning approach for identifying the cultural dimension. This approach detected clusters of ACO names based on the co-occurrence of terms (see the Supplementary 
Appendix). Informed by prior work on ACOs, we identified three clusters. The first, like our handcoded variable, comprised names that evoked the physician profession, using terms like "physician," "doctor," or "primary care." The second comprised names that were community focused, indicating an identification with a service area (e.g., "Accountable Care Coalition of Southeast Wisconsin”). The third cluster comprised names that reflected the organizational form of the ACO, using terms like "network," "alliance," or "coalition."

Because this approach yielded three clusters, we coded a new variable, $C^{*}$, where $C^{O^{*}}=1$ for the physician cluster (like our primary variable), $\mathrm{C}^{*}=2$ for the organizational cluster, and the reference category, $\mathrm{C}^{*}=0$, represented the community-focused cluster. Results using this alternative variable are similar to those of our main models. We still see a negative interaction between Structural disconnectedness and a culture of physician autonomy. However, the interpretation is different because the reference category is different.

Quality outcome measure. In Model 11, we run our main model using a dependent variable adjusted for quality. As discussed above, although quality is not the outcome used by CMS, CMS does use quality scores to calculate a "multiplier," which determines the proportion of shared savings that ACOs are eligible to receive if they achieve 2 or $3 \%$ cost savings. Therefore, we multiplied each ACO's Cost performance by the quality multiplier to create a quality-adjusted outcome. The results are similar to those in our main models. A different specification excluding the quality score control produced nearly identical results.

Dynamic panel. In Model 12, we assess the robustness of our results using a dynamic panel model (Arellano-Bond GMM), which additionally controls for past performance using further lags of the dependent variable as instruments after first-differencing to account for time-invariant fixed effects. As such, this approach includes historic performance as a covariate, which is important as ACOs may respond to past success or failure. We find that lagged Cost performance is indeed positively associated with the outcome (Table S4, Model 12: $\beta=0.48, p=0.045$ ), which suggests good performance in one year may be a predictor of good performance in the next. Coefficients for the interactions are similar to our main results; the interaction between Structural disconnectedness and Relational strength is 
negative (Table S4, Model 12: $\beta=-0.29, \mathrm{p}=0.006$ ) and the interaction between Structural disconnectedness and physician Cultural orientation is also similar to the main model (Table S4, Model 12: $\beta=-16.92, p=0.009)$. While our panel is relatively short, the consistency of our results using this instrumental variable approach helps alleviate some of our concerns about endogeneity.

Selection model. In Model 13, we estimate a selection model to account for ACOs' potentially "selecting into" a network approach. Our assumption is that ACOs use networks in some way, and that beneficiary-sharing is a good proxy for their structure. However, not all collaborative work is captured by beneficiary-sharing. Our main analysis includes ACOs with at least two members and one tie. 37 ACOs did not fit this criterion. Therefore, in the first stage, we predict "using a beneficiary-sharing network," with an exclusion restriction indicating whether the ACO spans multiple states. Because patient mobility is geographically localized, multi-state ACOs may be less likely to use networks, and may instead coordinate in other ways, such as sharing administrative resources. In the second stage, we predict Cost performance using our independent variables and their interactions. ${ }^{10}$ The results (shown in Model 16) are very similar to those of the main model. We report the first stage estimates in the Supplementary Appendix (Table S4).

Exclude outlier. In Model 14, we exclude an outlier ACO that reported negative performance of $44.25 \%$ relative to the benchmark in the first year of operation. This level of performance was considerably worse than the next lowest observed, $-15.76 \%$ relative to the benchmark. Excluding this ACO did not affect our results.

Exclude 2015. In Model 15, we exclude the last year of data from our panel (2015). The beneficiarysharing data that we utilized was truncated in 2015 by CMS to about $80 \%$ of the full timeframe (one year plus a sixty-day window). To account for the potential impact of this missing data, we exclude all ACO $\times$ year observations from 2015. Although the significance of the interaction terms is slightly diminished (as may be expected after dropping a year of data), the results are substantively supportive of our main findings.

\footnotetext{
10 The inverse Mills ratio in our second stage is not statistically significant, failing to reject the null. Therefore, the second stage results are the unadjusted output of the GLS regression, following Heckman (1979).
} 
Exclude noncontiguous states. Finally, in Model 16, we perform an additional analysis that excludes ACOs that span noncontiguous states (rather than simply controlling for them). This test is meant to assess whether our results are driven by ACOs which, because they span noncontiguous states, may not actually be trying to coordinate patient care. Excluding these ACOs does not change our results.

\section{DISCUSSION}

We began by observing that over the past few decades, network perspectives have become increasingly important in organizational research. However, research has tended mostly focus on how the embeddedness of actors within networks shapes their individual outcomes. Against this backdrop, a growing number of organizational researchers have undertaken a fundamental perspective shift, viewing networks more broadly, and concerning themselves with understanding whole network performance rather than that of the individual network actor. Findings suggest that the mechanisms and theoretical logic of whole networks may be systematically different from those of individual nodes (i.e., ego networks).

Drawing on classic work in macrostructural sociology, we developed a novel theoretical framework for understanding whole network performance that expands research on whole networks beyond a focus on structure to include two other fundamental—relational and cultural—properties or dimensions of whole networks. Inspired by research in biology, our whole network morphology framework suggests that the same whole network structures, when viewed together with different cultural and relational dimensions, may be associated with different behaviors, functions, and thus performance.

\section{Applying the Morphology Framework}

Empirical application of our framework to 250 interorganizational whole networks in the United States healthcare industry illustrates how the morphological perspective-specifically the interactions between structure and other whole network dimensions — can be operationalized in organizational settings. In addition, our analysis responds directly to calls for research on whole network performance in situ, where the complexity such structures can be better appreciated (Fang 
and Schilling 2010, Shore et al. 2015). Finally, by developing theory about the contingent relationships among whole networks' structural, relational, and cultural dimensions, we begin to reconcile conflicting findings about the structure-performance relationship.

Our findings from the ACO context supported a contingent view of the relationship between whole network structure and whole network performance that accounts for the relational and cultural dimensions. In keeping with existing research, we did not find a clear association between structural connectedness and whole network performance. However, we did find robust evidence that the relationship between structure and performance is dependent upon the relational strength and cultural orientation of the whole network. Specifically, we observed that structural disconnectedness is negatively associated with whole network performance at higher levels of relational strength, supporting our prediction that stronger ties among fragmented network partners may contribute to siloing that, in turn, likely hinders coordination. We also found a negative relationship between structural disconnectedness and whole network performance in ACOs that espoused a physician cultural orientation; however, this relationship flipped and was positive when disconnectedness was low (connectedness was high).

The finding that disconnectedness is associated with worse performance under a physician cultural orientation is interesting. One interpretation is that a culture of physician autonomy minimizes collective-oriented values, such as sacrificing for the success of the whole, which likely detracts from the collaborative ethos of ACOs. Since physicians are still reimbursed based on the work they perform locally, they may be resistant to changing their practices in a way that benefits collective outcomes, particularly when the cultural orientation of the whole network gives them license to be autonomous. As connectedness increases, and opportunities for contact and exposure among network members grow, it may be easier for an ACO to establish the buy-in necessary to generate a shared physician perspective. In contrast, the combination of low structural connectedness and a cultural orientation that predisposes members towards autonomy may create a siloing effect and therefore undermine the collaboration necessary for success.

\section{Limitations}


Our empirical findings should be viewed in the context of several limitations. The ACO is a relatively new organizational form, and therefore our data describe the characteristics of networks in their infancy. Thus, it is unclear if and to what extent the relationships we observe during these early stages will stabilize or change as they mature. Our use of observational data also limits our ability to make causal inferences, though consistency throughout additional tests and alternative specifications (Arellano-Bond dynamic panel and two-stage selection models) suggests that the observed relationships between the key dimensions of whole network morphology are quite robust. Finally, further work is needed to assess the utility of the morphology framework when applied to contexts where the desired network outcome is not collaboration.

Notwithstanding the idiosyncrasies of our context, we suggest that the interactions among structure and the relational and cultural dimensions of whole network morphology are fundamental features of all organizational networks. Although our empirical analysis was primarily meant to illustrate an application of our theoretical framework, our principal finding that the performance implications of structure are contingent upon other whole network dimensions is unlikely limited to healthcare.

\section{Implications and Future Work}

By switching to a whole network lens, we are afforded an opportunity to think beyond dyadic relationships to a larger canvas of social interaction. Specifically, our framework connects to observations Simmel made on social interaction in larger groups, where "a [member] can...hold the group responsible for what he has failed to do" (Simmel, [1950]: 134). Put differently, in large groups (i.e., three or more members) the relationship between each member and others in the collective can be more ambiguous. Consequently, social distance between members may be greater and accountability more diffuse.

In our study of ACOs, we theorized that this distance may manifest in reduced cooperation and coordination, in ways that may be connected to each of the whole network dimensions. Thus, members of strongly tied, but disconnected, whole networks may identify less with the collective and more with their local subgroup. Similarly, as alluded to earlier, a physician cultural orientation 
that values the autonomy of doctors over contributions to a collective goal may exacerbate the challenges of broader cooperation and coordination. Reducing the distance between members and the group by increasing connectedness may enable providers that value autonomy to develop a stronger sense of collective membership. In sum, by studying whole network performance, the social dynamic of non-dyadic groups becomes substantially more important relative to a focus on ego networks and the outcomes of individual nodes.

Of importance to practitioners is that the potential upside of whole networks may never be realized if member organizations fail to identify the potential pitfalls when entering into partnerships. Nevertheless, it may be difficult for participants, or architects, of organizational partnerships to take a whole network view of the structure, relations, or culture of a group partly because of complexity but also partly because of the contingent and emergent nature of outcomes. This difficulty may explain why many of the whole networks we studied exhibited considerable disconnectedness or possessed other curious features, such as being geographically distributed across noncontiguous states. Perhaps such ACOs identified other opportunities to collaborate or reduce costs locally or assumed that participants could more easily adapt to the new model of work despite challenges of distance or limited collaborative experience. Delving deeper into structurally disconnected ACOs may be informative for understanding their approach to achieving shared savings. Similarly, instances where members were able to achieve savings locally, but the network performed poorly as a whole, could provide a useful context to study externalities that may exist within collaboration networks.

Future work that attempts to unpack the interplay between formal and informal structures may also be valuable (McEvily et al. 2014). Certain variations in the whole network dimensions are "below the tip of iceberg" (Rosenkopf and Schleicher 2008), in the form of informal relationships. Informal interactions have featured prominently in network research (Podolny and Baron 1997). In our context, the formal network was defined by ACO membership, although informal relationships between entities that eventually formed the ACO existed prior to formalization. However, our study finds that these interactions may also be important for whole network performance after 
formalization. Thus, rather than being merely a means to a (formal) end, it may be important to consider whether informal ties not only predict formal ones, but are also shaped by them (Gulati and Puranam 2009). Indeed, considering that many collaborative efforts are created only to fail (Greve et al. 2010), there may be opportunities to develop new theories about how network formalization affects the underlying relationships and subsequent outcomes.

Finally, our research also connects to recent work on whole networks, such as Mahmood et al. (2016), which showed that the centralization of equity ties within business groups was associated with better performance of individual members. This relationship, however, was moderated by an external contingency - environmental turbulence. In contrast, our study proposes that such contingencies may be inherent to each network, in the relational and cultural dimensions. Future work may benefit from closer examination of the link between internal network dimensions and the external environment to develop deeper understandings of the dynamics and adaptability of whole networks.

\section{REFERENCES}

Ahuja G, Soda G, Zaheer A (2012) The Genesis and Dynamics of Organizational Networks. Organ. Sci. 23(2): 434-448.

Bail CA (2014) The cultural environment: Measuring culture with big data. Theory and Society, 43(3-4), 465-482.

Barkoczi D Galesic M (2016) Social Learning Strategies Modify the Effect of Network Structure on Group Performance. Nat. Commun. 7:13109.

Barnett ML, Landon BE, O’Malley AJ, Keating NL, Christakis NA (2011) “Mapping Physician Networks with Self-Reported and Administrative Data. Health Serv. Res, 46(5): 1592-1609.

Bavelas A (1950) Communication Patterns in Task-Oriented Groups. J. Acoust. Soc. Am, 22(6): 725730.

Beck DE, Margolin DA (2007) Physician Coding and Reimbursement. The Ochsner Joumal, 7(1):9-15. Berman SL, Down J, Hill CW (2002) Tacit Knowledge as A Source of Competitive Advantage in The National Basketball Association. Acad. Management J., 45(1): 13-31.

Berwick DM (2002) Escape Fire. New York: The Commonwealth Fund.

Berwick DM (2008) The Triple Aim: Care, Health, and Cost. Health Aff. 27(3): 759-769.

Blau PM (1960) Structural Effects. Am. Sociol. Rev. 25(2): 178-193.

Blau PM (1964) Exchange and Power in Social Life. John Wiley Sons.

Blau PM. Schwartz JE (1984) Cross-cutting Social Circles: Testing a Macrosociological Theory of Intergroup

Relations. Orlando: Academic.

Bonacich P (1987) Power and centrality: A family of measures. Amer. J. Sociol. 92(5), 1170-1182.

Borgatti SP (2006) Identifying Sets of Key Players in a Social Network. Computational and Mathematical Organization Theory, 12: 21-34.

Bourgeois FC, Olson KL, Mandl KD (2010) Patients Treated at Multiple Health Care Facilities. Arch Intern Med, 170(22): 1989-1995.

Brass DJ, Galaskiewicz J, Greve HR, Tsai W (2004) Taking stock of networks and organizations: A 
multilevel perspective. Acad. Management J. 47(6):795-817.

Buhr H, Owen-Smith J (2010) Networks as Institutional Support: Law Firm and Venture Capitalist

Relations and Regional Diversity in High-Technology IPOs. Pp. 95-126 in Institutions and

Entrepreneurship. Emerald Group Publishing Limited.

Burt RS (1992) Structural Holes: The Social Structure of Competition. Harvard University Press.

Centers for Medicare Medicaid Services (CMS), HHS, (2017) Medicare Program; Revisions to Payment Policies Under the Physician Fee Schedule and Other Revisions to Part B for CY 2018;

Medicare Shared Savings Program Requirements; and Medicare Diabetes Prevention Program. Final rule. Federal Register, 82(219): 52976-53371.

Centola D (2010) The Spread of Behavior in an Online Social Network Experiment. Science, 329(5996): 1194-1197.

Centola D (2015) The Social Origins of Networks and Diffusion. Amer. J. Sociol. 120(5): 1295-1338. Cerulo KA (1997) Identity Construction: New Issues, New Directions. Annu. Rev. Sociol., 23(1): 385409.

Christie LS, Luce RD, Macy J (1952) Communication and Learning in Task-Oriented Groups. Technical Report 231. Cambridge, MA: Research Laboratory of Electronics MI.T.

Cohen A (1974) Two-Dimensional Man: An Essay on The Anthropology of Power and Symbolism in Complex

Society. Routledge and Kegan Paul.

Corritore M, Goldberg A, Srivastava SB (2019) Duality in diversity: How intrapersonal and interpersonal cultural heterogeneity relate to firm performance. Administrative Science Quarterly, 0001839219844175.

Cross DA, Lin SC, Adler-Milstein J (2016) Assessing Payer Perspectives on Health Information Exchange J Am Med Inform Assoc 23:297-303.

Cubanski J, Neuman T, Griffin S, Damico A (2016) Medicare Spending at the End of Life: A Snapshot of Beneficiaries Who Died in 2014 and the Cost of Their Care. Kaiser Family Foundation Issue Brief. Retrieved November 30, 2017 (https://www.kff.org/medicare/issue-brief/medicarespending-at-the-end-of-life/).

Davis JA (1967) Clustering and Structural Balance in Graphs. Human Relations, 20(2): 181-187.

Derex M, Boyd R (2016) Partial Connectivity Increases Cultural Accumulation Within Groups. Proc. Natl. Acad. Sci. U.S.A, 113(11):2982-2987.

Doyle G, Goldberg A, Srivastava S, Frank MC (2017) Alignment at work: Using language to distinguish the internalization and self-regulation components of cultural fit in organizations. In Proceedings of the 55th Annual Meeting of the Association for Computational Linguistics (Volume 1: Long Papers), pp. 603-612.

Dyer, Jeffrey H, Nobeoka K (2000) Creating and managing a high-performance knowledge-sharing network: the Toyota case. Strategic Management J. 21(3): 345-367.

Enemark DP, McCubbins MD, Paturi R, Weller N (2011) Does More Connectivity Help Groups to Solve Social Problems? In Proceedings of the 12th ACM Conference on Electronic Commerce, ACM, 2011.

Enemark D, McCubbins MD. Weller N (2014) Knowledge and Networks: An Experimental Test of How Network Knowledge Affects Coordination. Social Networks, 36: 122-133.

Fang C, Lee J, Schilling MA (2010) Balancing Exploration and Exploitation Through Structural Design: The Isolation of Subgroups and Organizational Learning. Organ. Sci. 21(3):625-642.

Fleming L, King III, C, Juda AI (2007) Small Worlds and Regional Innovation. Organ. Sci. 18(6):938954.

Freidson E (1988) Profession of Medicine: A Study of The Sociology of Applied Knowledge. University of Chicago Press.

Friedberg MW, Hussey PS. Schneider EC (2010) Primary Care: A Critical Review of The Evidence on Quality and Costs of Health Care. Health Aff., 29(5): 766-772.

Funk RJ (2014) Making the Most of Where You Are: Geography, Networks, And Innovation in Organizations. Acad. Management J. 57(1):193-222.

Funk RJ, Owen-Smith J, Landon BE, Birkmeyer JD, Hollingsworth JM (2017) Identifying Natural Alignments Between Ambulatory Surgery Centers and Local Health Systems: Building Broader 
Communities of Surgical Care. Medical Care, 55(2), e9-e15.

Ghomrawi HM, Funk RJ, Parks ML, Owen-Smith J and Hollingsworth JM (2018) Physician referral patterns and racial disparities in total hip replacement: A network analysis approach. PloS One, 13(2): e0193014.

Glynn MA. Abzug R (2002) Institutionalizing Identity: Symbolic Isomorphism and Organizational Names. Acad. Management J., 45(1):267-280.

Granovetter MS (1973) The Strength of Weak Ties. Amer. J. Sociol. 78(6):1360-1380.

Granovetter M (1985) Economic Action and Social Structure: The Problem of Embeddedness. Amer. J. Sociol. 91(3): 481-510.

Granqvist N, Grodal S, Woolley JL (2013) Hedging your bets: Explaining executives' market labeling strategies in nanotechnology. Organ. Sci., 24(2), 395-413.

Greve HR, Baum JAC, Mitsuhashi H, Rowley TJ (2010) Built to Last but Falling Apart: Cohesion, Friction, And Withdrawal from Interfirm Alliances. Acad. Management J. 53(2):302-322.

Grossmann I, Varnum, ME (2015) Social structure, infectious diseases, disasters, secularism, and cultural change in America. Psychological Science, 26(3), 311-324.

Guetzkow H, Simon HA (1955) The Impact of Certain Communication Nets Upon Organization and Performance in Task-Oriented Groups. Manag. Sci. 1(3-4):233-250.

Gulati R, Wohlgezogen F Zhelyazkov P (2012) The Two Facets of Collaboration: Cooperation and Coordination in Strategic Alliances. Acad. Management A. 6(1): 531-583.

Gulati R Puranam P (2009) Renewal Through Reorganization: The Value of Inconsistencies

Between Formal and Informal Organization. Organ. Sci., 20(2):422-440.

Guler I, Nerkar A (2012) The Impact of Global and Local Cohesion on Innovation in The

Pharmaceutical Industry. Strategic Management J. 33(5):535-549.

Hansen MT (1999) The Search-Transfer Problem: The Role of Weak Ties in Sharing Knowledge

Across Organization Subunits. Admin. Sci. Quart. 44(1): 82-111.

Hatch MJ. Schultz M (2002) The Dynamics of Organizational Identity. Human Relations, 55(8):989-

1018.

Heidl RA, Steensma HK, Phelps C (2014) Divisive Faultlines And the Unplanned Dissolutions of Multipartner Alliances. Organ. Sci. 25(5):1351-1371.

Hollingsworth JM, Funk RJ, Garrison SA, Owen-Smith J, Kaufman SR, Landon BE. Birkmeyer JD. 2015a. Differences between physician social networks for cardiac surgery serving communities with high versus low proportions of black residents. Medical Care, 53(2):160-167.

Hollingsworth JM, Funk RJ, Owen-Smith J, Landon BE, Hollenbeck BK, Birkmeyer JD. 2015b.

Assessing the Reach of Health Reform to Outpatient Surgery with Social Network Analysis. Annals of Surgery, 261(3): 468-472.

Horn E Jacobi J (2006) The Critical Care Clinical Pharmacist: Evolution of an Essential Team Member. Critical Care Medicine, 34(3): S46-S51.

Ibarra H (1993) Personal networks of women and minorities in management: A conceptual framework. Acad. Management R. 18(1):56-87.

Iwashyna TJ, Christie JD, Moody J, Kahn JM, Asch DA (2009) The Structure of Critical Care Transfer Networks. Medical Care, 47(7): 787-793.

Jackson JC, Gelfand M, De S, Fox A (2019) The loosening of American culture over 200 years is associated with a creativity-order trade-off. Nature Human Behaviour, 3(3): 244-250.

Kaiser Family Foundation. (1996) Profile of Medicare Beneficiaries by Race and Ethnicity: A

Chartpack. Retrieved November 21, 2017 (https://www.kff.org/medicare/report/profile-ofmedicare-beneficiaries-by-race-and-ethnicity-a-chartpack/).

Kearns M, Suri S, Montfort N (2006) An Experimental Study of the Coloring Problem on Human Subject Networks. Science, 313: 824-827.

Kelly C Kelly J (1994) Who Gets Involved in Collective Action? Social Psychological Determinants of Individual Participation in Trade Unions. Human Relations, 47(1):63-88.

King BG, Felin T, Whetten DA (2010) Perspective-Finding the organization in organizational theory: A meta-theory of the organization as a social actor. Organ. Sci. 21(1):290-305. 
King BG (2011) The tactical disruptiveness of social movements: Sources of market and mediated disruption in corporate boycotts. Social Problems, 58(4), 491-517.

King BG, Clemens ES, Fry M (2011) Identity Realization and Organizational Forms: Differentiation and

Consolidation of Identities Among Arizona's Charter Schools. Organ. Sci. 22(3):554-572.

Kogut B and Zander U (1996) What Firms Do? Coordination, Identity, And Learning. Organ.

Sci., 7(5):502-518.

Koehl MA.R (1996) When Does Morphology Matter? Annual Review of Ecology and

Systematics, 27(1):501-542.

Kozlowski AC, Taddy M, Evans JA (2019) The Geometry of Culture: Analyzing the Meanings of Class through Word Embeddings. American Sociological Review, 84(5):905-49.

Kreindler SA, Larson BK, Wu FM, Carluzzo KL, Gbemudu JN, Struthers A, Van Citters AD, Shortell SS, Nelson EC, Fisher. E. S (2012) Interpretations of Integration in Early Accountable Care Organizations. Milbank Quarterly, 90(3): 457-483.

Landon BE, Keating NL, Barnett ML, Onnela J, Paul S, O’Malley AJ, Keegan T, Christakis NA (2012) Variation in Patient-Sharing Networks of Physicians Across the United States. JAMA, 308(3): 265-273.

Lassman D, Hartman M, Washington B, Andrews K, Catlin A (2014) US Health Spending Trends by Age and Gender: Selected Years 2002-10. Health Aff. 33(5): 815-822.

Lau DC, Murnighan JK (1998) Demographic Diversity and Faultlines: The Compositional Dynamics of Organizational Groups. Acad. Management R. 23(2), 325-340.

Lazer D, Friedman A (2007) The Network Structure of Exploration and Exploitation. Admin. Sci. Quart. 52(1995):667-694.

Levin DZ. Cross R (2004) The Strength of Weak Ties You Can Trust: The Mediating Role of Trust in Effective Knowledge Transfer. Manag. Sci., 50(11):1477-1490.

Light D, Levine S (1988) The changing character of the medical profession: a theoretical overview. Milbank Q. 66(Suppl 2):10-32.

Macy Jr J, Christie LS. Luce RD (1953) Coding Noise in a Task-oriented Group. The Journal of Abnormal and Social Psychology, 48(3): 401-409.

Mason WA, Jones A Goldstone RL (2008) Propagation of Innovations in Networked Groups. Journal of Experimental Psychology: General, 137(3): 422-433.

Mason W, Watts DJ (2012) Collaborative Learning in Networks. Proc. Natl. Acad. Sci. U.S.A, 109(3):764-769.

McCubbins MD, Paturi R, Weller N (2009) Connected Coordination: Network Structure and Group Coordination. American Politics Research, 37(5):899-920.

McEvily B, Soda G Tortoriello M (2014) More Formally: Rediscovering the Missing Link Between

Formal Organization and Informal Social Structure. Acad. Management A., 8(1):299-345.

McFarland DA, Moody J, Diehl D, Smith JA, Thomas RJ (2014) Network Ecology and Adolescent Social Structure. Am. Sociol. Rev., 79(6), 1088-1121.

Milo R, Shen-Orr S, Itzkovitz S, Kashtan N, Chklovskii D Alon U (2002) Network motifs: simple building blocks of complex networks. Science, 298(5594): 824-827.

Moody J, White DR (2003) Structural Cohesion and Embeddedness: A Hierarchical Concept of Social Groups. Am. Sociol. Rev. 68(1): 103-127.

Moran P (2005) Structural vs. Relational Embeddedness: Social Capital and Managerial Performance. Strategic Management J. 26: 1129-1151.

Mulder M (1960) Communication Structure, Decision Structure and Group Performance. Sociometry, 23(1):1-14.

Nahapiet J Ghoshal S (1998) Social Capital, Intellectual Capital, and the Organizational Advantage. Acad. Management R., 23(2):242-266.

Okhuysen GA. Bechky BA (2009) Coordination in Organizations: An Integrative Perspective. Acad. Management A. 3(1): 463-502.

Okie S (2012) The Evolving Primary Care Physician. N. Engl. J. Med, 366(20): 1849-1853. 
Oyserman D, Lee SW (2008) Does culture influence what and how we think? Effects of priming individualism and collectivism. Psychol. Bull. 134(2):311.

Pachucki MA, Breiger RL (2010) Cultural holes: Beyond relationality in social networks and culture. Annu. Rev. Sociol., 36, 205-224.

Paruchuri S (2010) Intraorganizational Networks, Interorganizational Networks, and the Impact of Central Inventors: A Longitudinal Study of Pharmaceutical Firms. Organ. Sci. 21(1): 63-80.

Paruchuri S, Awate S (2017) Organizational Knowledge Networks and Local Search: The Role of Intra-Organizational Inventor Networks. Strategic Management J. 38: 657-675.

Pettigrew AM (1979) On Studying Organizational Cultures Andrew M. Pettigrew. Admin. Sci. Quart., 24(4):570-581.

Podolny JM. Baron JN (1997) Resources and Relationships: Social Networks and Mobility in The Workplace. Am. Sociol. Rev. 62(5):673-693.

Polidoro Jr F, Ahuja G Mitchell W (2011) When the social structure overshadows competitive incentives: The effects of network embeddedness on joint venture dissolution. Acad. Management J., 54(1):203-223.

Portes A, Sensenbrenner J (1993) Embeddedness and Immigration: Notes on the Social

Determinants of Economic Action. Amer. J. Sociol. 98:1320-50.

Porter MF (1980) An Algorithm for Suffix Stripping. Program, 14(3): 130-137.

Powell WW, Koput KW, Smith-Doerr L, Owen-Smith J (1999) Network Position and Firm

Performance: Organizational Returns to Collaboration in the Biotechnology Industry. Research in the Sociology of Organizations 16(1):129-59.

Provan KG, Milward HB (1995) A Preliminary Theory of Interorganizational Network

Effectiveness: A Comparative Study of Four Community Mental Health Systems. Admin. Sci. Quart. 41(1):1-33.

Provan KG, Sebastian JG (1998) Networks Within Networks: Service Link Overlap, Organizational Cliques, And Network Effectiveness. Acad. Management J. 41(4): 453-463.

Reagans R, Argote L, Brooks D (2005) Individual Experience and Experience Working Together: Predicting Learning Rates from Knowing Who Knows What and Knowing How to Work Together. Manag. Sci. 51(6): 869-881.

Rickert J (2012) Patient-Centered Care: What It Means and How to Get There. Health Aff. Blog, 24.

Ritzer G Walczak D (1988) Rationalization and the Deprofessionalization Of Physicians. Social

Forces, 67(1):1-22.

Rosenkopf L, Schleicher T (2008) Below the Tip of The Iceberg: The Co-Evolution of Formal and Informal Interorganizational Relations in The Wireless Telecommunications Industry. Managerial and Decision Economics, 29(5):425-441.

Rowley T, Behrens D Krackhardt D (2000) Redundant Governance Structures: An Analysis of Structural and Relational Embeddedness in The Steel and Semiconductor Industries. Strategic Management J. 21(3): 369-386.

Seibert SE, Kraimer ML, Liden RC (2001) A Social Capital Theory of Career Success. Acad. Management J. 44(2): 219-237.

Shirado H, Christakis NA (2017) Locally Noisy Autonomous Agents Improve Global Human Coordination in Network Experiments. Nature, 545(7654):370-374.

Shore J, Bernstein E, Lazer D (2015) Facts and Figuring: An Experimental Investigation of Network Structure and Performance in Information and Solution Spaces. Organ. Sci. 26(5):1-15.

Simmel G (1950) The Sociology of Georg Simmel. Trans. K. H. Wolff. Glencoe, IL: Free Press.

Soda G, Usai A, Zaheer A (2004) Network Memory: The Influence of Past and Current Networks on Performance. Acad. Management J. 47(6): 893-906.

Srivastava SB, Goldberg A (2017) Language as a Window into Culture. California Management Review, 60(1):56-69.

Srivastava SB, Goldberg A, Manian VG, Potts C (2018) Enculturation trajectories: Language, cultural adaptation, and individual outcomes in organizations. Management Science, 64(3):1348-64.

Starr P (2008) The Social Transformation of American Medicine: The Rise of a Sovereign Profession and The 
Making of a Vast Industry. Basic books.

Stevens R 1998. American Medicine and the Public Interest: Updated Edition with a New Introduction. Univ of California Press.

Tocqueville AD (2000) Democracy in America. (1835) Trans. Harvey C. Mansfield and Delba Winthrop. Chicago: University of Chicago Press.

Uzzi B (1997) Social structure and competition in interfirm networks: The paradox of embeddedness. Admin. Sci. Quart. 42(1): 35-67.

Vasudeva G, Zaheer A, Hernandez E (2013) The Embeddedness of Networks: Institutions, Structural Holes, And Innovativeness in The Fuel Cell Industry. Organ. Sci. 24(3): 645-663.

Vogus TJ, Sutcliffe KM. Weick KE (2010) Doing No Harm: Enabling, Enacting, and Elaborating a Culture of Safety in Health Care. Academy of Management Perspectives, 24(4):60-77.

Wagner EH, Austin BT, Von Korff M (1996) Organizing Care for Patients with Chronic Illness. Milbank Q., 74(4): 511-544.

Wheeler JA (1990) Information, Physics, Quantum: The Search for Links. Complexity, Entropy, and the Physicians of Information. Westview.

Zollo M, Reuer JJ, Singh H (2002) Interorganizational Routines and Performance in Strategic Alliances. Organ. Sci. 13(6): 701-713. 
TABLE 1

Whole Network Morphology Framework

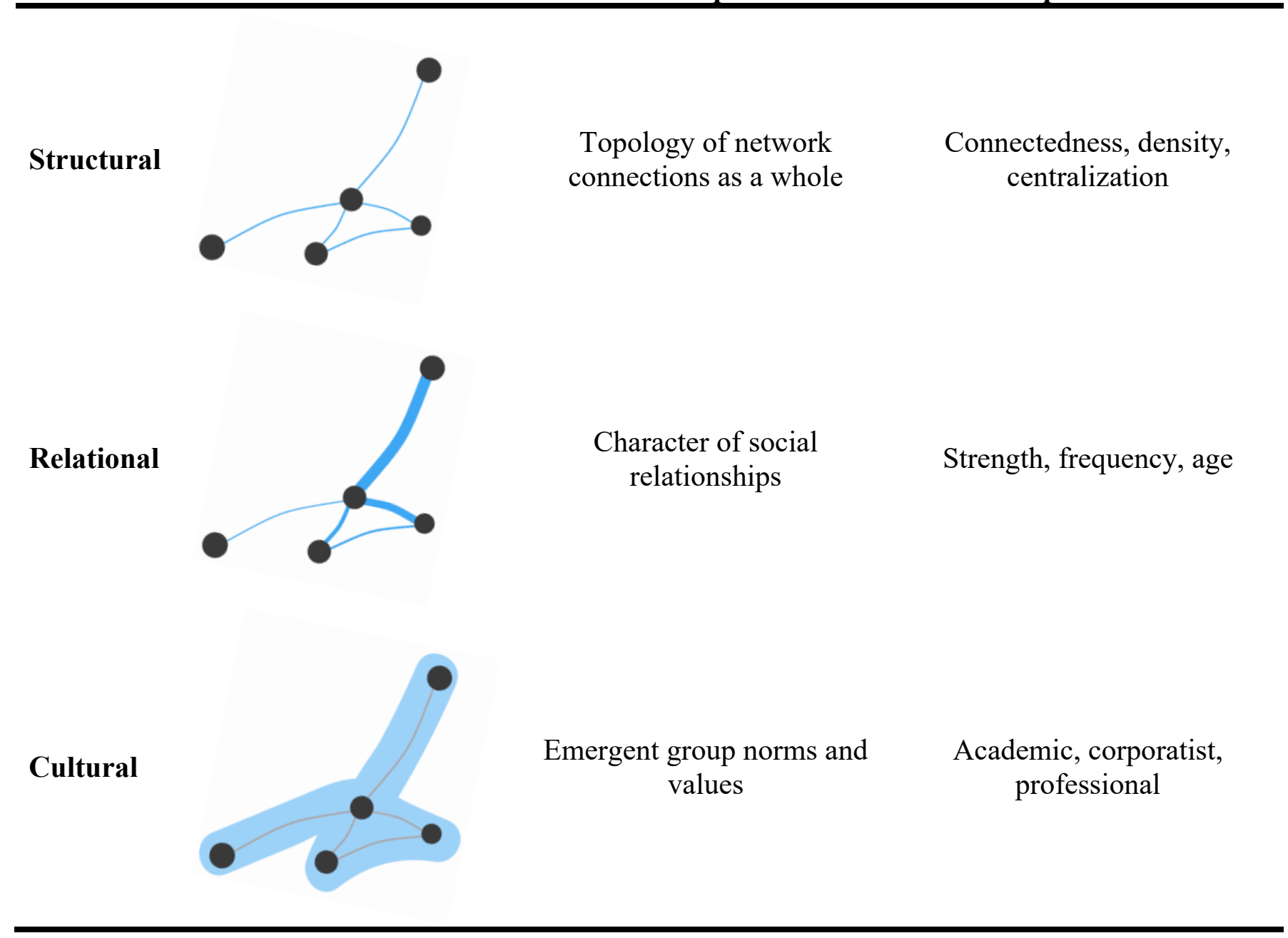


TABLE 2

OLS Regression Models of Cost Performance

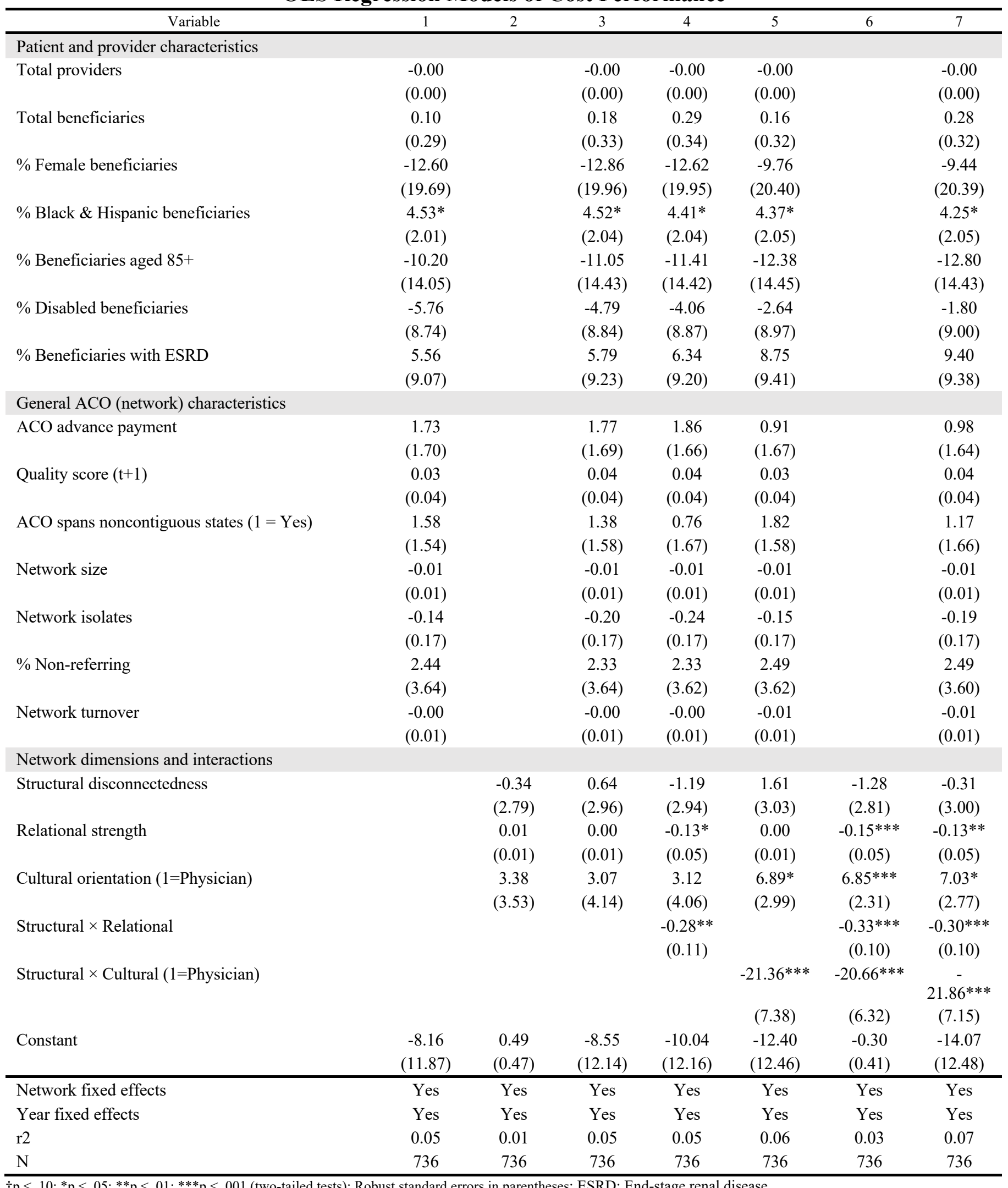


FIGURE 1

Shifting Levels of Analysis: Ego to Whole Network

Ego Network View 1

Whole Network View

\section{Ego Network View 2}

O
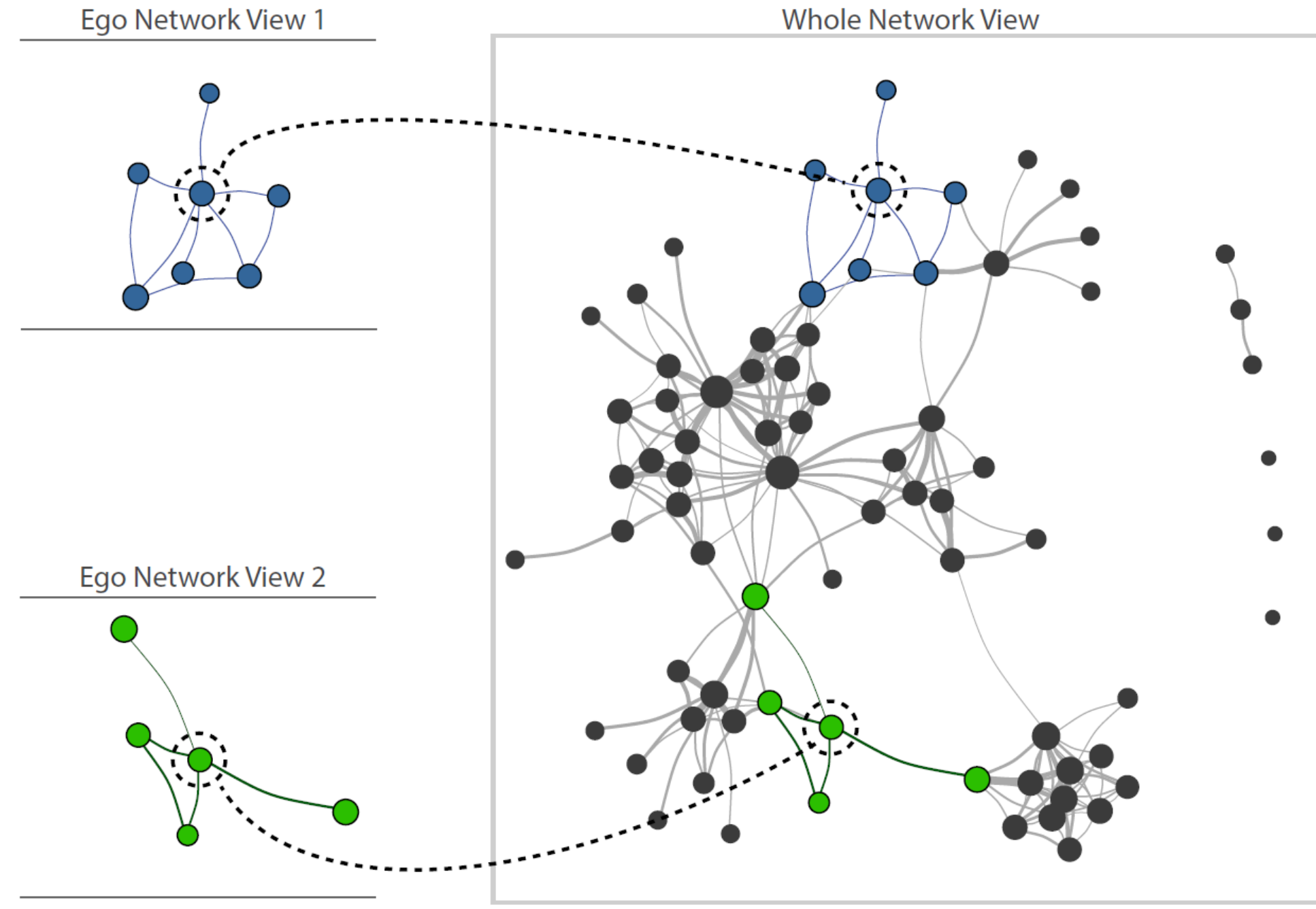

Ego networks may be very different from the broader networks that they are part of, and the performance of one ego network may not necessarily align with that of other ego networks, or the network as a whole. Moreover, theories developed to explain behavior and performance at the ego level do not necessarily translate to the higher level, highlighting the need for whole network level theorizing. 
FIGURE 2

Illustrative Whole Network Dimensions for Two ACOs

ACO A

More Connected, Greater Relational Strength, Physician Orientation

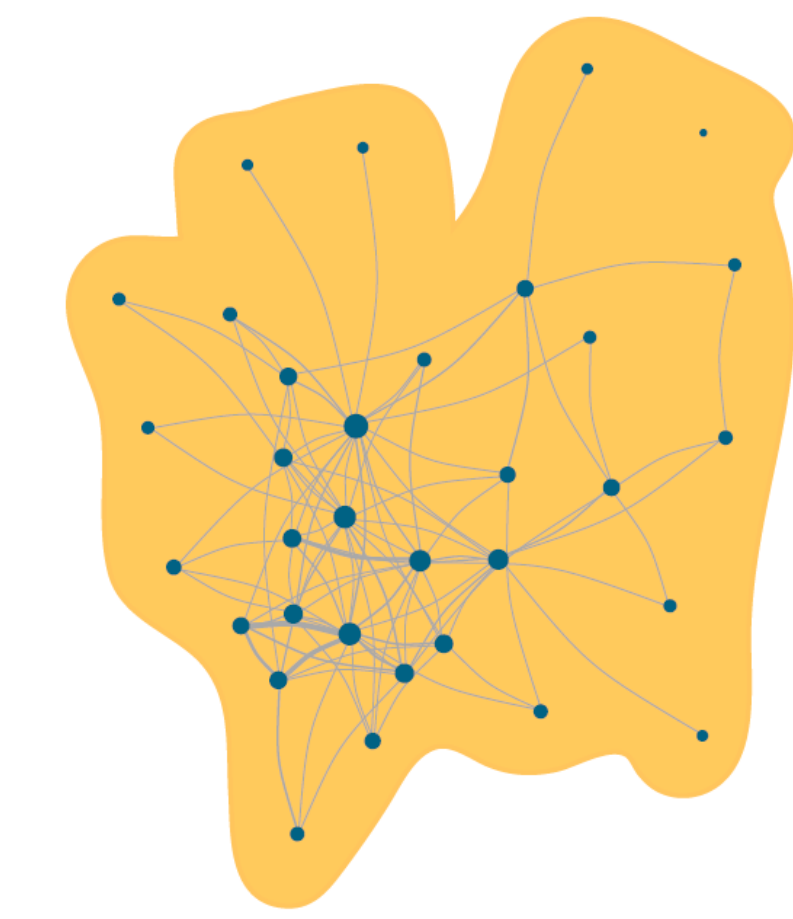

ACO B

More Disconnected, Lower Relational Strength, Other Orientation

- ACO member organization

_ Beneficiary-sharing tie

Physician orientation

Other orientation

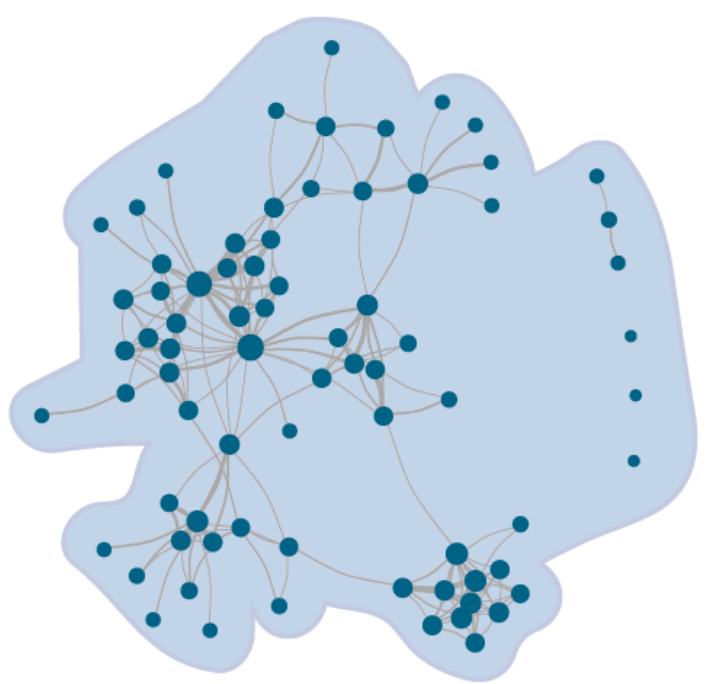

Two ACOs, exhibiting different degrees of structural disconnectedness and relational strength, and different cultural orientations. Node size reflects relative prominence of the organization in the network based on the number of connections to other network members, line thickness reflects relational strength. ACO A, which was coded as having a physician cultural orientation, is more structurally connected and has greater relational strength than ACO B, which did not have a culture of physician autonomy. 
FIGURE 3

Predicted Values of Cost Performance at Different Levels of Structural Disconnectedness and Relational Strength (Left), and Structural Disconnectedness and Cultural Orientation (Right)
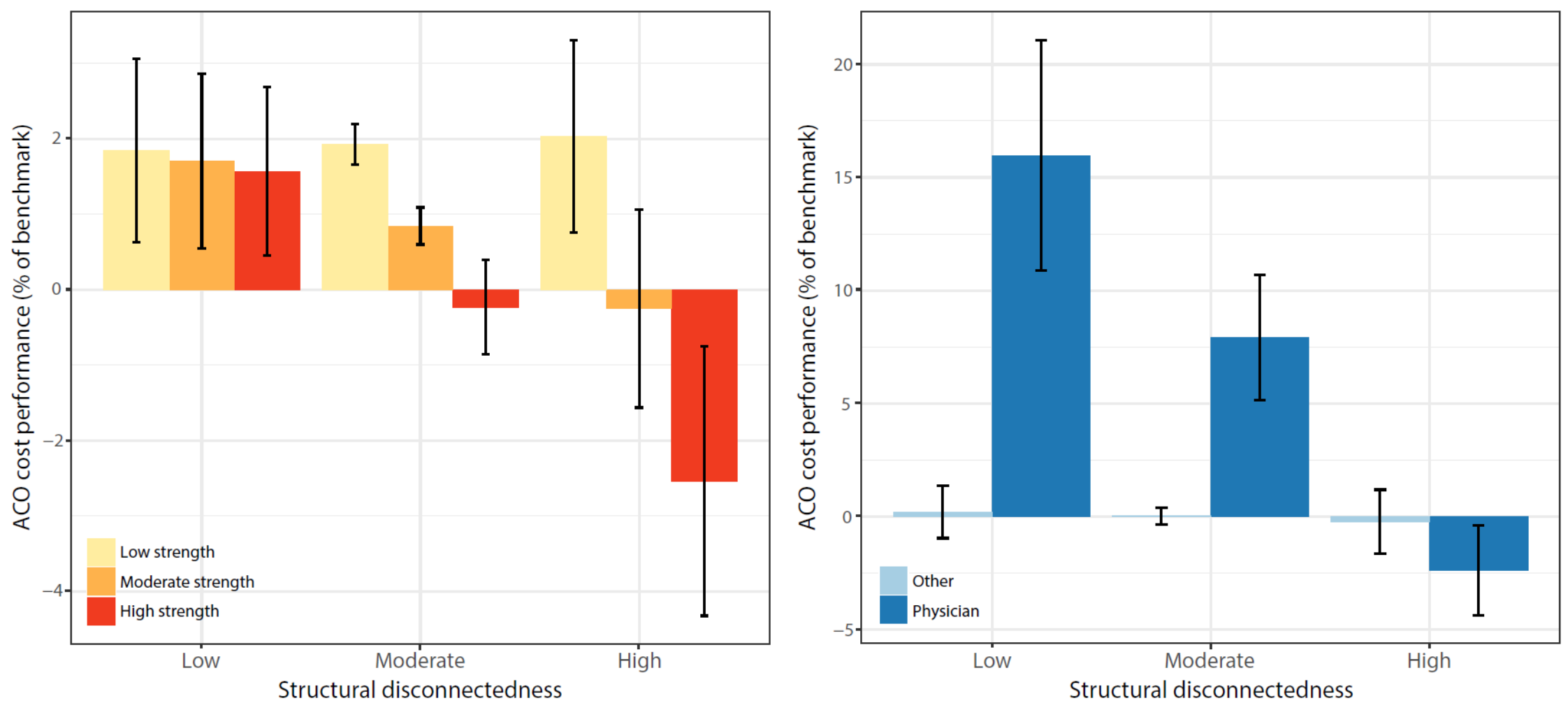

Predicted values of ACO cost performance (based on Model 7, Table 2) in terms of the percentage savings relative to the benchmark, illustrating the interaction between structural disconnectedness and relational strength at low, moderate, and high levels of each variable. Most ACOs perform relatively well at low

disconnectedness. However, as disconnectedness increases, increasing relational strength is associated with considerably worse outcomes. Low disconnectedness represents the $10^{\text {th }}$ percentile, moderate the median, and high the $90^{\text {th }}$ percentile. Low, moderate, and high levels of relational strength also represent the $10^{\text {th }}, 50^{\text {th }}$, and $90^{\text {th }}$ percentile values, respectively. Standard error bars are shown. 


\section{APPENDIX A. ADDITIONAL NOTES ON NETWORK CONSTRUCTION}

The Medicare Shared Savings Program Accountable Care Organization (ACO) Research Identifiable Files (RIFs) are made available by Medicare for each performance year, beginning with ACOs that started in 2012 and 2013. These files provide participant information for ACOs, including the organizations - identified by tax identification numbers (TINs) — and the associated physicians identified by national provider identifier (NPI) number. Physicians are listed individually and nested within the organizational TINs, which, according to the initial ACO rules, can only be assigned to one ACO. ${ }^{1}$ We use this nested association between NPIs and TINs to assign providers to organizations.

Although there is predominantly 1:1:1 assignment between physicians, organizations, and ACOs, we discovered that about $5 \%$ of physicians were associated with more than one ACO, and about $17 \%$ of physicians were associated with more than one TIN. We attributed these overlapping physicians to the first organization and/or ACO with which they were affiliated in the Provider RIF (which is randomly sorted). Each year, approximately $11 \%$ of the TINs (670 in 2012, 2,577 in 2015) were not associated with any physicians. Investigating these TINs, we found that many represented legal businesses for individual physicians that were associated with other ACO member organizations. Others included healthcare organizations, such as diagnostic labs or home care, which would not be expected to have patient sharing ties. There were also several TINs that were not identifiable based on the legal business name of the entity. Finally, a small number $(\sim 2 \%)$ of such TINs were associated with hospitals or clinics. The lack of associated NPIs may indicate that these organizations, or some part of them, were participating in a similar capacity to non-referring organizations (e.g., ancillary services, non-billable capabilities) that would not require specific physician NPIs to be listed. We cannot map networks based on organizational TINs without associated physician NPIs; therefore, organizations without affiliated NPIs do not appear in the beneficiary-sharing networks. However, we control for their presence in the analyses by including a variable for disconnected organizations.

\footnotetext{
${ }^{1}$ For more information, see the November 15, 2017 Federal Register (https://www.gpo.gov/fdsys/pkg/FR-2017-1115/pdf/2017-23953.pdf). Notwithstanding these rules, it appears that overlap in TINs across ACOs may be increasingly common and CMS intends to make accommodations to try to account for these.
} 


\section{APPENDIX B. HAND CODING CULTURAL ORIENTATION}

Two coders independently coded a list of 697 unique terms that appeared in the names of ACOs that were operating from 2013-2015. The coders focused on classifying terms as having or not having a clear connection to physicians. A list of the top twenty most frequently appearing terms can be found in Table S1 below.

Table S1. Top twenty most frequent terms in ACO names (2013-2015)

\begin{tabular}{ll}
\hline LLC & MEDICAL \\
ACO & HEALTHCARE \\
CARE & COALITION \\
HEALTH & PHYSICIAN \\
ACCOUNTABLE & COLLABORATIVE \\
NETWORK & RURAL \\
PARTNERS & QUALITY \\
INC & CLINIC \\
ALLIANCE & COMMUNITY \\
PHYSICIANS & PRIMARY
\end{tabular}

Once each term was coded, each coders' determinations were compared and reconciled. If no agreement could be reached, a third person was brought in to resolve the dispute.

Codes were then aggregated to the ACO name level, with each name associated with a score reflecting the relative number of "physician" and "other" terms. Most names would be coded as representing a physician orientation if the name contained more physician coded versus other coded terms. Exceptions were made, however, if the name contained at least one term that was deemed to be unambiguously physician-oriented. These terms were "physician," "doctor," and "md." Names containing at least one of these terms were coded as physician orientation $\left(C^{O}=1\right)$. A sample of coded ACO names can be found in Table S2. A full list of coded names is available upon request.

Table S2. Illustrative Coded ACO names

\begin{tabular}{l|l}
\hline \multicolumn{1}{c|}{$\boldsymbol{C} \mathbf{O}=\mathbf{0}$ (Other orientation) } & \multicolumn{1}{c}{$\boldsymbol{C} \boldsymbol{O}=\mathbf{1}$ (Physician orientation) } \\
\hline Matrix ACO LLC & Care is Primary ACO LLC \\
Lower Shore ACO, LLC & $\begin{array}{l}\text { Affiliated Physicians Medical Group ACO, Inc. } \\
\text { Primary Care Alliance LLC }\end{array}$ \\
Integrated Care Alliance, LLC & St. Luke's Clinic Coordinated Care, Ltd. \\
Keep Well ACO LLC & WakeMed Key Community Care, LLC \\
OneHealth Nebraska ACO, LLC & Commonwealth Primary Care ACO \\
Arkansas Health Network LLC & Alabama Physician Network, LLC \\
KentuckyOne Health Partners, LLC & Physician Performance Network of Arizona, LLC \\
WellStar Health Network, LLC &
\end{tabular}




\section{APPENDIX C. MACHINE LEARNING APPRAOCH TO CODING CULTURAL ORIENTATION}

To complement our hand coding approach to creating the $C^{\circ}$ (cultural orientation) variable, we also used a machine learning technique. In our main methodology, based on prior writing on ACOs and health care organizations more generally, we were primarily interested in determining the presence or absence of a strong physician orientation based on the content of each ACO's name. However, for the machine learning approach, we were also interested in seeing what additional clusters, if any, would be detected using an established clustering methodology.

We began with the same cleaned and standardized (i.e., punctuation removed, common abbreviations replaced) list of ACO names $(\mathrm{N}=551)$ used for hand coding. This list was then used to construct a large [name $\times$ term] matrix, in which rows indexed each unique (cleaned, standardized) ACO name, columns indexed each unique (cleaned, standardized) term appearing in the complete list of those names, and cell entries corresponded to the frequency with which the focal term appeared within the focal name. From this name $\times$ term matrix, we then created a new, square, symmetric matrix, in which rows and columns both indexed unique ACO names, and cell entries corresponded to the Euclidean distance between focal rows and columns based on the co-occurrence of terms. Finally, using this matrix of distances, we then clustered ACO names using Ward's (1963) hierarchical clustering algorithm.

The next step in the process was to understand the meaningful differences between the identified clusters (see dendrogram of clusters below in Figure S2). We used our contextual knowledge and drew on studies from health policy (e.g., Fisher \& Shortell, 2012; Shortell et al., 2014) to identify three, contextually meaningful clusters of ACO names. The first comprised names that were more community or geography-focused, indicating a clear identification with the particular region they were serving. The second comprised names that were strongly connected to the physician profession, using terms such as "physician," "doctor," or "primary care," which are clearly associated with medical doctors. The third cluster comprised names that reflected the organizational or bureaucratic form of the ACO, and that used terms such as "network," "alliance," or "coalition."

These clusters—physician-oriented, community-oriented, and organizational—aligned well with descriptive studies of ACOs. The first, physician-oriented, seems to match ACOs which predominantly comprise private practices and physician groups (e.g., UCLA Faculty Practice Group). These ACOs often do not include a hospital or similar large, multispecialty organization (Shortell et al., 2014). The second, community-oriented, aligns with ACOs that place a greater emphasis on local communities (e.g., Piedmont Community Health Collaborative) or the health of specific populations (e.g., Asian American Accountable Care Organization). The third, organizational, is more closely aligned with ACOs that have been described as being more corporate or administrative (Kreindler et al., 2012; Shortell et al., 2014), and tend to be closely associated with larger healthcare systems that are more likely to employ physicians, rather than contract with independent physician groups. 
Figure S1. Dendrogram of ACO name clusters

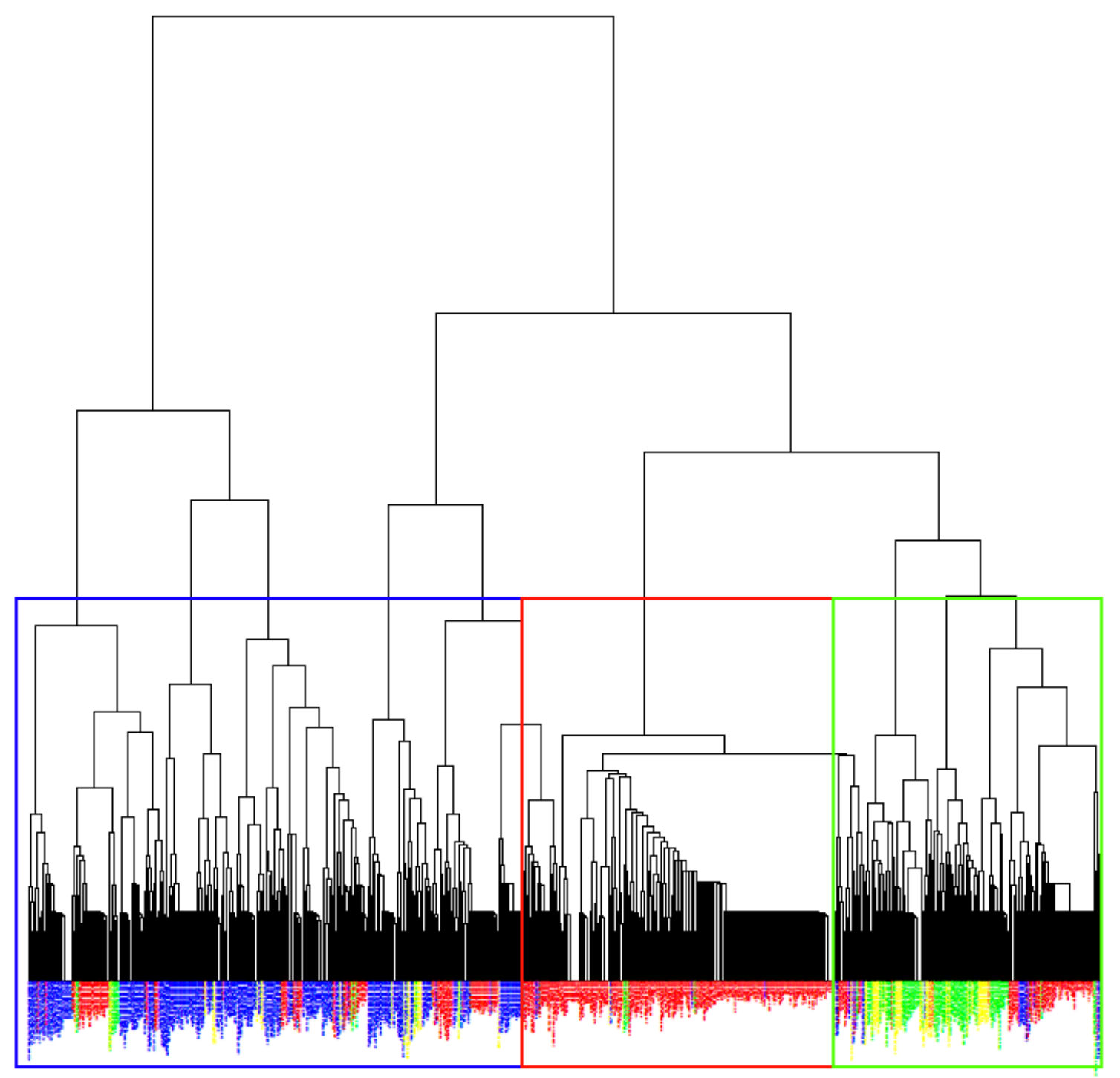

The hierarchical clustering algorithm grouped ACO names according to similarity. Based on these results, we identified three major clusters of cultural orientation: (1) organizational or bureaucratic orientation, (2) physician orientation, and (3) community orientation. The specific ACO names at the bottom-most row of the dendogram are not visible due to the large size of the image. A high-resolution version of the figure can be provided upon request. 


\section{APPENDIX D. VALIDATION OF CULTURAL ORIENTATION MEASURE}

To evaluate the face validity of our measure of ACO cultural orientation, we extracted the complete text of ACO websites (when available) for networks in our sample. We then determined the rate, per 1,000 words, at which words appeared in the ACO website text for physician oriented and nonphysician oriented ACOs (based on our hand-coding methodology). After cleaning the list to identify substantive descriptive words and removing technical or nonsense terms (e.g., html, http, link, sitemap) we calculated, for each word, the ratio of the frequency of appearance on physician-oriented websites to the frequency of appearance on non-physician oriented sites.

Below is a graphical representation of the top- 20 and bottom- 20 words in terms relative frequency. That is, words on the top, with the yellow bars, are those that appeared more frequently on physician-oriented websites. Words on the bottom, with the blue bars, are those that appeared more frequently on non-physician-oriented websites. The full, cleaned list of words is available upon request. See the main text for more discussion of the figure.

Figure S2. Top words associated with physician oriented vs. non-physician oriented ACO websites.

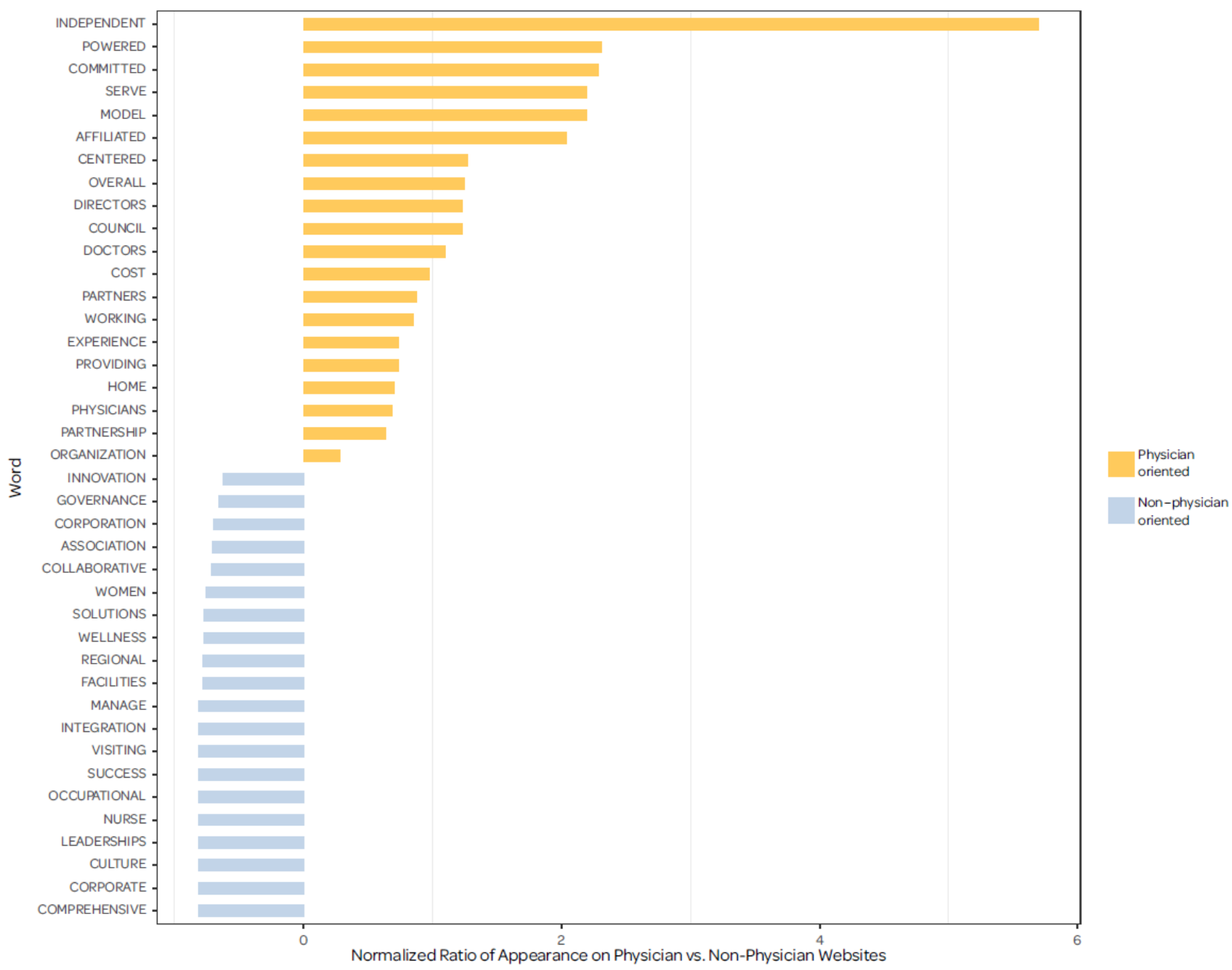


APPENDIX E. DESCRIPTIVE STATISTICS

TABLE S3

Descriptive Statistics and Pairwise Correlations

\begin{tabular}{|c|c|c|c|c|c|c|c|c|c|c|}
\hline Variable & $\mathrm{N}$ & Mean & S.D. & 1 & 2 & 3 & 4 & 5 & 6 & 7 \\
\hline 1. Cost performance & 736 & 1.17 & 5.89 & & & & & & & \\
\hline 2. Structural disconnectedness & 736 & -0.01 & 0.27 & $0.18^{*}$ & & & & & & \\
\hline 3. Relational strength & 736 & 0.71 & 23.87 & $-0.14^{*}$ & $-0.40^{*}$ & & & & & \\
\hline 4. Cultural orientation ( $1=$ Physician) & 736 & 0.10 & 0.29 & 0.06 & $0.15^{*}$ & $-0.10^{*}$ & & & & \\
\hline 5. Total providers & 736 & 563.24 & 753.32 & $-0.13^{*}$ & $-0.21 *$ & $0.31 *$ & 0.03 & & & \\
\hline 6. Total beneficiaries & 736 & 1.71 & 1.7 & $-0.08^{*}$ & $-0.07 *$ & $0.21 *$ & 0.07 & $0.73 *$ & & \\
\hline 7. $\%$ Female beneficiaries & 736 & 0.48 & 0.17 & 0.01 & 0.06 & -0.04 & -0.01 & -0.01 & $0.17 *$ & \\
\hline 8. $\%$ Black \& Hispanic beneficiaries & 736 & 0.39 & 0.49 & 0.05 & -0.02 & 0.02 & -0.00 & 0.00 & $-0.20^{*}$ & $-0.94 *$ \\
\hline 9. $\%$ Beneficiaries aged $85+$ & 736 & 0.19 & 0.12 & 0.02 & -0.04 & 0.02 & 0.02 & -0.04 & $-0.17 *$ & $-0.92 *$ \\
\hline 10. $\%$ Disabled beneficiaries & 736 & 0.11 & 0.08 & -0.01 & $0.08^{*}$ & -0.04 & -0.06 & 0.04 & 0.02 & $0.64^{*}$ \\
\hline 11. \% Beneficiaries with ESRD & 736 & 0.34 & 0.59 & 0.01 & -0.05 & 0.03 & 0.00 & 0.00 & $-0.18^{*}$ & $-0.99 *$ \\
\hline 12. ACO advance payment & 736 & 0.09 & 0.29 & $0.14 *$ & $0.24 *$ & $-0.11 *$ & $0.12 *$ & $-0.21 *$ & $-0.17^{*}$ & $-0.09 *$ \\
\hline 13. Quality score $(t+1)$ & 736 & 90.79 & 8.39 & 0.07 & $-0.13^{*}$ & 0.06 & 0.02 & $0.12 *$ & $0.19 *$ & $0.32 *$ \\
\hline 14. ACO spans noncontiguous states $(1=\mathrm{Yes})$ & 736 & 0.05 & 0.21 & 0.02 & $0.23^{*}$ & -0.06 & $0.08^{*}$ & 0.06 & $0.10^{*}$ & 0.03 \\
\hline 15. Network size & 736 & 44.41 & 55.7 & 0.04 & $0.25^{*}$ & $-0.12^{*}$ & $0.18^{*}$ & $0.40 *$ & $0.47 *$ & 0.03 \\
\hline 16. Network isolates & 736 & 1.50 & 2.66 & $0.13^{*}$ & $0.71 *$ & $-0.21 *$ & $0.18^{*}$ & -0.05 & $0.08 *$ & 0.05 \\
\hline 17. $\%$ Non-referring & 736 & 0.38 & 0.22 & $0.12^{*}$ & $0.42^{*}$ & $-0.15^{*}$ & -0.01 & -0.04 & -0.02 & $0.14^{*}$ \\
\hline \multirow[t]{2}{*}{ 18. Network turnover } & 736 & -0.20 & 10.73 & -0.03 & -0.02 & 0.01 & -0.01 & 0.06 & $0.09 *$ & $0.13^{*}$ \\
\hline & 8 & 9 & 10 & 11 & 12 & 13 & 14 & 15 & 16 & 17 \\
\hline 9. $\%$ Beneficiaries aged $85+$ & $0.91 *$ & & & & & & & & & \\
\hline 10. $\%$ Disabled beneficiaries & $-0.57 *$ & $-0.72 *$ & & & & & & & & \\
\hline 11. $\%$ Beneficiaries with end-stage renal disease & $0.96^{*}$ & $0.95^{*}$ & $-0.66^{*}$ & & & & & & & \\
\hline 12. ACO advance payment & $0.09 *$ & $0.09 *$ & -0.07 & $0.08^{*}$ & & & & & & \\
\hline 13. Quality score $(t+1)$ & $-0.30^{*}$ & $-0.31^{*}$ & $0.20^{*}$ & $-0.32 *$ & -0.04 & & & & & \\
\hline 14. ACO spans noncontiguous states $(1=$ Yes $)$ & -0.03 & -0.02 & $0.09 *$ & -0.03 & $0.08^{*}$ & 0.01 & & & & \\
\hline 15. Network size & -0.03 & 0.03 & -0.04 & -0.02 & $-0.13^{*}$ & $-0.08^{*}$ & $0.10^{*}$ & & & \\
\hline 16. Network isolates & -0.02 & -0.06 & $0.10^{*}$ & -0.05 & $0.16^{*}$ & -0.07 & $0.27 *$ & $0.27 *$ & & \\
\hline 17. $\%$ Non-referring & $-0.14^{*}$ & -0.07 & 0.00 & $-0.13 *$ & 0.03 & $-0.14^{*}$ & $0.09 *$ & $0.30 *$ & $0.31 *$ & \\
\hline 18. Network turnover & $-0.12^{*}$ & $-0.12 *$ & 0.07 & $-0.14 *$ & -0.01 & 0.07 & 0.06 & $0.08 *$ & 0.01 & -0.02 \\
\hline
\end{tabular}

$* \mathrm{p}<0.05$; ESRD: End-stage renal disease 
APPENDIX F. ROBUSTNESS TESTS

Table S4. Robustness Tests

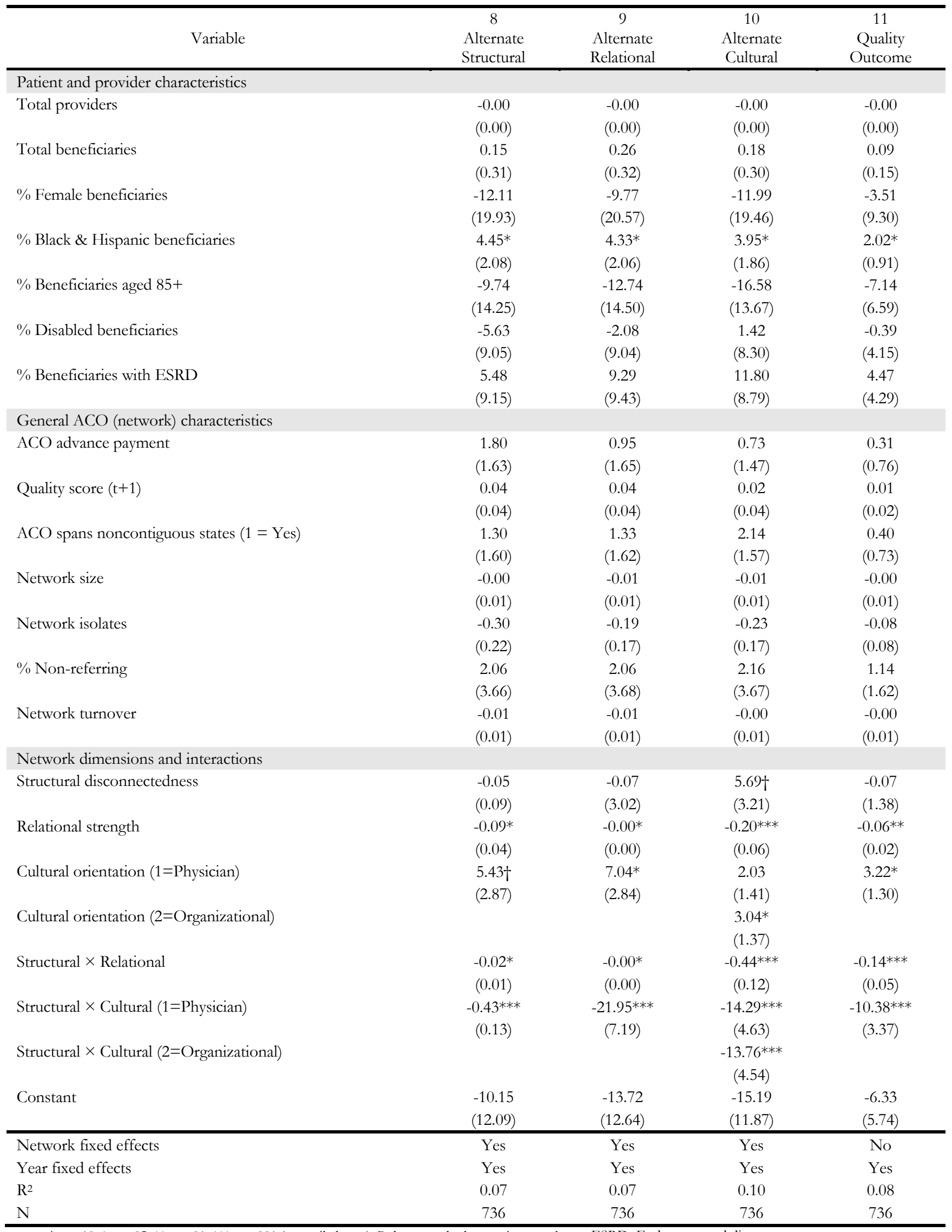

tp $<.10 ; *_{\mathrm{p}}<.05 ; * * \mathrm{p}<.01 ; * * * \mathrm{p}<.001$ (two-tailed tests); Robust standard errors in parentheses; ESRD: End-stage renal disease 
Table S4. Robustness Tests (Continued)

\begin{tabular}{|c|c|c|c|c|c|}
\hline Variable & $\begin{array}{c}12 \\
\text { Dynamic } \\
\text { Panel }\end{array}$ & $\begin{array}{c}13 \\
\text { Selection } \\
\text { Model }\end{array}$ & $\begin{array}{c}14 \\
\text { Exclude } \\
\text { Outlier }\end{array}$ & $\begin{array}{c}15 \\
\text { Exclude } 2015\end{array}$ & $\begin{array}{c}16 \\
\text { Exclude } \\
\text { Noncontiguous }\end{array}$ \\
\hline \multicolumn{6}{|l|}{ Patient and provider characteristics } \\
\hline Total providers & $\begin{array}{l}-0.00 \\
(0.00)\end{array}$ & $\begin{array}{l}-0.00 \dagger \\
(0.00)\end{array}$ & $\begin{array}{l}-0.00 \\
(0.00)\end{array}$ & $\begin{array}{l}-0.00 \\
(0.00)\end{array}$ & $\begin{array}{l}-0.00 \\
(0.00)\end{array}$ \\
\hline Total beneficiaries & $\begin{array}{c}0.30 \\
(0.36)\end{array}$ & $\begin{array}{c}0.29 \\
(0.24)\end{array}$ & $\begin{array}{c}0.28 \\
(0.32)\end{array}$ & $\begin{array}{c}0.15 \\
(0.49)\end{array}$ & $\begin{array}{c}0.24 \\
(0.33)\end{array}$ \\
\hline$\%$ Female beneficiaries & $\begin{array}{l}-16.70 \\
(34.51)\end{array}$ & $\begin{array}{c}-9.48 \\
(15.39)\end{array}$ & $\begin{array}{c}-8.01 \\
(20.93)\end{array}$ & $\begin{array}{c}5.96 \\
(14.31)\end{array}$ & $\begin{array}{l}-10.20 \\
(21.16)\end{array}$ \\
\hline$\%$ Black \& Hispanic beneficiaries & $\begin{array}{c}3.97 \\
(2.86)\end{array}$ & $\begin{array}{l}4.24^{*} \\
(1.89)\end{array}$ & $\begin{array}{l}4.20 * \\
(2.07)\end{array}$ & $\begin{array}{c}2.10 \\
(1.78)\end{array}$ & $\begin{array}{l}4.19 \dagger \\
(2.15)\end{array}$ \\
\hline$\%$ Beneficiaries aged $85+$ & $\begin{array}{c}-6.04 \\
(15.53)\end{array}$ & $\begin{array}{l}-13.01 \\
(10.99)\end{array}$ & $\begin{array}{l}-13.08 \\
(14.43)\end{array}$ & $\begin{array}{l}-11.33 \\
(15.49)\end{array}$ & $\begin{array}{l}-15.00 \\
(15.31)\end{array}$ \\
\hline$\%$ Disabled beneficiaries & $\begin{array}{l}-14.88 \\
(10.49)\end{array}$ & $\begin{array}{l}-1.76 \\
(7.19)\end{array}$ & $\begin{array}{l}-2.09 \\
(8.98)\end{array}$ & $\begin{array}{l}-0.73 \\
(9.61)\end{array}$ & $\begin{array}{l}-0.97 \\
(9.45)\end{array}$ \\
\hline$\%$ Beneficiaries with ESRD & $\begin{array}{c}-7.78 \\
(12.10)\end{array}$ & $\begin{array}{c}9.47 \\
(7.60)\end{array}$ & $\begin{array}{c}9.18 \\
(9.38)\end{array}$ & $\begin{array}{l}12.48 \\
(9.84)\end{array}$ & $\begin{array}{l}10.29 \\
(9.73)\end{array}$ \\
\hline \multicolumn{6}{|l|}{ General ACO (network) characteristics } \\
\hline ACO advance payment & $\begin{array}{l}-1.95 \\
(2.78)\end{array}$ & $\begin{array}{c}0.98 \\
(1.26)\end{array}$ & $\begin{array}{c}1.04 \\
(1.66)\end{array}$ & & $\begin{array}{c}0.98 \\
(1.66)\end{array}$ \\
\hline Quality score $(t+1)$ & $\begin{array}{c}0.04 \\
(0.04)\end{array}$ & $\begin{array}{c}0.04 \\
(0.03)\end{array}$ & $\begin{array}{c}0.04 \\
(0.04)\end{array}$ & $\begin{array}{c}0.02 \\
(0.04)\end{array}$ & $\begin{array}{c}0.03 \\
(0.04)\end{array}$ \\
\hline ACO spans noncontiguous states $(1=$ Yes $)$ & $\begin{array}{l}-2.94 \\
(2.63)\end{array}$ & & $\begin{array}{c}1.15 \\
(1.65)\end{array}$ & & \\
\hline Network size & $\begin{array}{l}-0.00 \\
(0.01)\end{array}$ & $\begin{array}{l}-0.01 \\
(0.01)\end{array}$ & $\begin{array}{l}-0.01 \\
(0.01)\end{array}$ & $\begin{array}{l}-0.01 \\
(0.01)\end{array}$ & $\begin{array}{l}-0.00 \\
(0.01)\end{array}$ \\
\hline Network isolates & $\begin{array}{l}-0.23 \\
(0.19)\end{array}$ & $\begin{array}{l}-0.20 \\
(0.13)\end{array}$ & $\begin{array}{l}-0.19 \\
(0.17)\end{array}$ & $\begin{array}{l}-0.09 \\
(0.26)\end{array}$ & $\begin{array}{l}-0.18 \\
(0.20)\end{array}$ \\
\hline$\%$ Non-referring & $\begin{array}{c}0.60 \\
(4.61)\end{array}$ & $\begin{array}{c}2.50 \\
(2.61)\end{array}$ & $\begin{array}{c}2.45 \\
(3.61)\end{array}$ & $\begin{array}{c}0.02 \\
(2.47)\end{array}$ & $\begin{array}{c}2.34 \\
(3.77)\end{array}$ \\
\hline Network turnover & $\begin{array}{l}-0.00 \\
(0.02)\end{array}$ & $\begin{array}{l}-0.01 \\
(0.01)\end{array}$ & $\begin{array}{l}-0.01 \\
(0.01)\end{array}$ & $\begin{array}{l}-0.01 \\
(0.02)\end{array}$ & $\begin{array}{l}-0.01 \\
(0.01)\end{array}$ \\
\hline Network dimensions and interactions & & & & & \\
\hline Structural disconnectedness & $\begin{array}{l}-2.97 \\
(3.69)\end{array}$ & $\begin{array}{l}-0.33 \\
(2.24)\end{array}$ & $\begin{array}{l}-0.45 \\
(3.01)\end{array}$ & $\begin{array}{l}-0.20 \\
(3.31)\end{array}$ & $\begin{array}{l}-0.58 \\
(3.10)\end{array}$ \\
\hline Relational strength & $\begin{array}{c}-0.13^{* *} \\
(0.05)\end{array}$ & $\begin{array}{c}-0.14^{* * *} \\
(0.04)\end{array}$ & $\begin{array}{c}-0.13^{* *} \\
(0.05)\end{array}$ & $\begin{array}{l}-0.04 \\
(0.08)\end{array}$ & $\begin{array}{c}-0.13^{*} \\
(0.05)\end{array}$ \\
\hline Cultural orientation ( $1=$ Physician $)$ & $\begin{array}{l}7.70 \dagger \\
(4.48)\end{array}$ & $\begin{array}{c}7.04 * * * \\
(2.11)\end{array}$ & $\begin{array}{l}7.04 * \\
(2.77)\end{array}$ & $\begin{array}{c}6.49 \\
(5.11)\end{array}$ & $\begin{array}{l}6.86^{*} \\
(2.92)\end{array}$ \\
\hline Structural $\times$ Relational & $\begin{array}{c}-0.29 * * \\
(0.10)\end{array}$ & $\begin{array}{c}-0.30^{* * *} \\
(0.08)\end{array}$ & $\begin{array}{c}-0.29 * * * \\
(0.10)\end{array}$ & $\begin{array}{l}-0.19 \\
(0.13)\end{array}$ & $\begin{array}{c}-0.30 * * \\
(0.11)\end{array}$ \\
\hline Structural $\times$ Cultural $(1=$ Physician $)$ & $\begin{array}{c}-16.92 \dagger \\
(10.06)\end{array}$ & $\begin{array}{c}-21.77 * * * \\
(5.53)\end{array}$ & $\begin{array}{c}-21.73^{* * *} \\
(7.12)\end{array}$ & $\begin{array}{c}-22.78^{*} \\
(11.28)\end{array}$ & $\begin{array}{c}-20.09 * * \\
(7.56)\end{array}$ \\
\hline Past performance $(\mathrm{t})$ & $\begin{array}{l}0.48^{*} \\
(0.24)\end{array}$ & & & & \\
\hline$\varrho$ & & $\begin{array}{l}-0.01 \\
(0.08)\end{array}$ & & & \\
\hline Constant & $\begin{array}{c}8.32 \\
(20.03)\end{array}$ & $\begin{array}{l}-13.74 \\
(14.94)\end{array}$ & $\begin{array}{l}-13.87 \\
(12.51)\end{array}$ & $\begin{array}{l}-16.03 \\
(11.22)\end{array}$ & $\begin{array}{l}-14.36 \\
(12.76)\end{array}$ \\
\hline ACO fixed effects & $\mathrm{No}$ & Yes & Yes & Yes & Yes \\
\hline Year fixed effects & No & Yes & Yes & Yes & Yes \\
\hline $\mathrm{R}^{2}$ or pseudo- $\mathrm{R}^{2}$ & 0.18 & 0.77 & 0.07 & 0.11 & 0.07 \\
\hline $\mathrm{N}$ & 374 & 839 & 734 & 439 & 702 \\
\hline
\end{tabular}

tp $<.10 ;{ }^{*} \mathrm{p}<.05 ;{ }^{* *} \mathrm{p}<.01 ;{ }^{* * *} \mathrm{p}<.001$ (two-tailed tests); Robust standard errors in parentheses; pseudo-R ${ }^{2}$ reported for Models $15 \& 16$ 
Table S5. Selection model (Model 13), first and second stages

\begin{tabular}{|c|c|c|}
\hline Variable & $\begin{array}{c}\text { First stage Probit, "Is } \\
\text { a Network" }\end{array}$ & Second stage OLS \\
\hline \multicolumn{3}{|l|}{ Patient and provider characteristics } \\
\hline Total providers & $\begin{array}{c}0.00^{* * *} \\
(0.00)\end{array}$ & $\begin{array}{c}-0.00 \dagger \\
(0.00)\end{array}$ \\
\hline Total beneficiaries & $\begin{array}{l}-2.18 \\
(1.52)\end{array}$ & $\begin{array}{c}0.28 \\
(0.24)\end{array}$ \\
\hline$\%$ Female beneficiaries & $\begin{array}{c}3.11 \\
(2.74)\end{array}$ & $\begin{array}{c}-9.45 \\
(15.38)\end{array}$ \\
\hline$\%$ Black \& Hispanic beneficiaries & $\begin{array}{l}-0.13 \\
(0.59)\end{array}$ & $\begin{array}{l}4.25^{*} \\
(1.89)\end{array}$ \\
\hline$\%$ Beneficiaries aged $85+$ & $\begin{array}{c}1.97 \\
(1.40)\end{array}$ & $\begin{array}{l}-12.80 \\
(11.03)\end{array}$ \\
\hline$\%$ Disabled beneficiaries & $\begin{array}{l}2.49 * \\
(1.17)\end{array}$ & $\begin{array}{l}-1.81 \\
(7.21)\end{array}$ \\
\hline$\%$ Beneficiaries with ESRD & $\begin{array}{c}0.71 \\
(1.21)\end{array}$ & $\begin{array}{c}9.40 \\
(7.62)\end{array}$ \\
\hline \multicolumn{3}{|l|}{ General ACO (network) characteristics } \\
\hline ACO advance payment & & $\begin{array}{c}0.98 \\
(1.26)\end{array}$ \\
\hline Quality score $(\mathrm{t}+1)$ & $\begin{array}{c}-0.03 * * * \\
(0.01)\end{array}$ & $\begin{array}{c}0.04 \\
(0.03)\end{array}$ \\
\hline ACO spans noncontiguous states ( $1=$ Yes $)$ & $\begin{array}{c}4.93^{* * * *} \\
(0.10)\end{array}$ & $\begin{array}{c}1.17 \\
(1.24)\end{array}$ \\
\hline Network size & & $\begin{array}{l}-0.01 \\
(0.01)\end{array}$ \\
\hline Network isolates & & $\begin{array}{l}-0.19 \\
(0.13)\end{array}$ \\
\hline$\%$ Non-referring & & $\begin{array}{l}2.49 \\
(2.62)\end{array}$ \\
\hline Network turnover & & $\begin{array}{l}-0.01 \\
(0.01)\end{array}$ \\
\hline \multicolumn{3}{|l|}{ Network dimensions and interactions } \\
\hline Structural disconnectedness & & $\begin{array}{l}-0.31 \\
(2.24)\end{array}$ \\
\hline Relational strength & & $\begin{array}{c}-0.13 * * * \\
(0.04)\end{array}$ \\
\hline Cultural orientation ( $1=$ Physician $)$ & & $\begin{array}{c}7.03^{* * * *} \\
(2.12)\end{array}$ \\
\hline Structural $\times$ Relational & & $\begin{array}{c}-0.30^{* * * *} \\
(0.08)\end{array}$ \\
\hline Structural $\times$ Cultural $(1=$ Physician $)$ & & $\begin{array}{c}-21.87 * * * \\
(5.54)\end{array}$ \\
\hline$\varrho$ & $\begin{array}{l}-0.00 \\
(0.08)\end{array}$ & - \\
\hline Constant & $\begin{array}{c}3.32 * * * \\
(0.93)\end{array}$ & $\begin{array}{l}-13.49 \\
(15.02)\end{array}$ \\
\hline ACO fixed effects & No & Yes \\
\hline Year fixed effects & No & Yes \\
\hline $\mathrm{R}^{2}$ or pseudo- $\mathrm{R}^{2}$ & & 0.77 \\
\hline $\mathrm{N}$ & 839 & 736 \\
\hline
\end{tabular}

tp $<.10 ;{ }^{*} \mathrm{p}<.05 ;{ }^{* *} \mathrm{p}<.01 ;{ }^{* * *} \mathrm{p}<.001$ (two-tailed tests) 